\title{
MEASURABLE SELECTION FOR PURELY ATOMIC GAMES
}

\author{
ZIV HELLMAN \\ Department of Economics, Bar Ilan University \\ YEHUDA JOHN LEVY \\ Adam Smith Business School, University of Glasgow
}

\begin{abstract}
A general selection theorem is presented constructing a measurable mapping from a state space to a parameter space under the assumption that the state space can be decomposed as a collection of countable equivalence classes under a smooth equivalence relation. It is then shown how this selection theorem can be used as a general purpose tool for proving the existence of measurable equilibria in broad classes of several branches of games when an appropriate smoothness condition holds, including Bayesian games with atomic knowledge spaces, stochastic games with countable orbits, and graphical games of countable degree-examples of a subclass of games with uncountable state spaces that we term purely atomic games. Applications to repeated games with symmetric incomplete information and acceptable bets are also presented.
\end{abstract} tion.

KEYWORDS: Bayesian games, stochastic games, graphical games, measurable selec-

\section{INTRODUCTION}

COUNTER-EXAMPLES serve to refute conjectures, challenge assumptions, and sharpen concepts. Their negativity often spurs new questions. Having ruled out some result due to the existence of a counter-example, one can subsequently ask: what conditions or assumptions would suffice for positive results instead? It may also happen that initially disparate counter-examples taken together point to a unifying underlying phenomenon.

Here we make use of similarities amongst a series of recent counter-examples to obtain equilibrium existence results in several types of games with uncountable state spaces. We also introduce a new sub-class of games with uncountable state spaces, which we term purely atomic games, ${ }^{1}$ that forms the natural framework for our results, with much potential for further research.

Striking counter-examples to the existence of measurable equilibria, in different branches of game theory, have been discovered in recent years. In stochastic games, Levy (2013b) presented an example of a stochastic game that has no measurable stationary equilibria, even if the game consists of deterministic transitions. This requires an uncountable state space; previous results, going back to the 1960s and 1970s, had established the existence of stationary equilibria in games with finite and countable state spaces, and it was long conjectured that this would extend to the uncountable case until Levy's counter-example settled the matter. (Levy and McLennan (2015) presented an additional counter-example to this conjecture, albeit in a class in which transitions are not purely atomic; hence the subject matter of that paper falls outside of the type of structures discussed in this paper.)

Ziv Hellman: ziv.hellman@biu.ac.il

Yehuda John Levy: john.levy@glasgow.ac.uk

Ziv Hellman acknowledges research support by Israel Science Foundation Grant 1626/18.

${ }^{1}$ Not to be confused with the terms non-atomic and atomic games, which appear in the literature in relation to certain types of cooperative games. 
In Bayesian games, Simon (2003) presented an example of a three-player Bayesian game over an uncountable state space that admits no measurable Bayesian equilibrium. This was further enhanced by an example in Hellman (2014b) of a two-player Bayesian game that admits no measurable ${ }^{2}$ Bayesian $\varepsilon$-equilibrium for sufficiently small $\varepsilon$. More recently, Simon and Tomkowicz (2018) have constructed an example of a three-player Bayesian game that admits no measurable ex ante $\varepsilon$-equilibrium. This also required uncountable state spaces: the existence of both ex ante and Bayesian equilibria in games with finite state spaces is a classic result of Harsanyi (1967), which was extended to countable games by Simon (2003).

Lehrer and Samet (2011) presented an example of a knowledge space, based on an example in Simon (2000), over which there is a measurable common prior yet over each individual common knowledge component no common prior exists. Hellman and Levy (2017) contains a similar knowledge space in which, in each common knowledge component, players can find an acceptable bet (or trade) to which they agree, but, over the entire space, the players must agree to disagree as there exists no measurable mutually agreeable bet.

We have here a series of counter-examples in different sub-fields of game theory arising from cross-inspirations in several directions. ${ }^{3}$ This naturally raises the following questions: Can we find conditions that turn these negative results into positive results on equilibrium existence? Furthermore, given the history of the discoveries of these counter-examples, is it possible to find common elements to all of them, and if so, can the understanding of the common elements be of use in fashioning a unified proof method for positive results?

We show in this paper that the answer to these questions is yes. First of all, note that all the above counter-examples are modeled on games with uncountable state spaces (indeed, this was necessary, as in each case, the results in the finite and countable cases had previously been settled). Moreover, with the notable exception of Simon and Tomkowicz (2018), they also all share the characteristic of being given to decomposition into disjoint countable equivalence classes; in the Bayesian games, for example, the decomposition is given by countable common knowledge components, while in the stochastic games, the orbit of each state, under the action of the stochastic game transition function, is countable.

This leads us to define a new sub-class of games with uncountable state spaces. Informally, a purely atomic game is a game whose underlying state space can be divided into countable equivalence classes, such that those equivalence classes are 'natural' structures for their respective game types. Formalizing this idea requires specifying what is meant by 'underlying state space' and 'natural equivalence structures' for particular types of games, which we accomplish here for Bayesian, stochastic, and graphical games.

For Bayesian games, the natural equivalence relation concept is the common knowledge relation on the underlying knowledge space. A purely atomic Bayesian game is then one in which every common knowledge component is countable. The natural equivalence structure in stochastic games is a countable orbit: if, from each state $q$, there are at most countably many subsequent states under the transition functions and at most countably many previous states that could lead to $q$, then one can define an equivalence relation

\footnotetext{
${ }^{2}$ There is a relatively short step from the non-existence of a measurable equilibrium to an example of a game without any equilibria at all, measurable or not. Details on this appear in Section 4 of Hellman (2014b).

${ }^{3}$ Tracing out some of the cross-inspirations: Simon and Tomkowicz (2018) acknowledged ideas from Hellman (2014b), which was inspired by studying Levy (2013a), which in turn was inspired by Simon (2003). Hellman and Levy (2017) followed the lead of an example in Lehrer and Samet (2011), which in turn was inspired by Simon (2000).
} 
of states under transition with countable equivalence classes. Calling these equivalence classes orbits, a purely atomic stochastic game is one whose orbits are all countable. (Repeated games of symmetric incomplete information can naturally be modeled in this framework, following Neyman and Sorin (2003, Chapter 21).) In graphical games, the nodes of a graph are the players of the game, and the payoff of a player depends only on his actions and those of his neighbors in the graph. ${ }^{4}$ The natural equivalence structures for this game are the connected components of the graph.

We note here that games with a continuum of states that also satisfy the purely atomic property described above can arise in a variety of models. In Example 4.5 of Section 4.2, we show how misspecification of exchange rates can lead to uncertainty amongst agents regarding the true value of a product, resulting in a purely atomic structure. Section 5.3 presents an example of agents who place repeated double-or-nothing bets (Example 5.4), again yielding a purely atomic example. These examples show that there are naturally arising situations that satisfy a regularity property we term smoothness, which will be absolutely key for our results.

Smoothness was a key element in the measurable Bayesian equilibrium existence theorem of Hellman and Levy (2017). The proof of the theorem there consisted of slicing the state space into non-disjoint, countable common knowledge equivalence classes, each of which admits a Bayesian equilibrium in the game restricted to that equivalence class, and then 'gluing' together one selected equilibrium from each class to form a single measurable equilibrium over the entire space. The process requires a mechanism for selecting an equilibrium from each class in just the right way to enable the final glued product to be measurable.

In this paper, that idea is generalized to a 'one size fits all' general purpose theorem that can apply to all smooth purely atomic games. The main element is the measurable selection theorem (Theorem 3.1), which makes use of infinitary logic to provide a general schema for the task of selecting equilibrium for each equivalence class of a smooth countable equivalence relation and gluing them into a measurable global equilibrium.

Once the general proof schema is at hand, adaptation to particular types of games is straightforward. For virtually any type of game, if equilibria exist in the countable case, then, by application of the measurable selection proof schema, measurable equilibria exist in the uncountable case for smooth purely atomic games. Other applications of the technique we present include equilibrium existence in certain repeated games with symmetric incomplete information, the construction of acceptable bets in Bayesian games, as well as some approximation techniques which we in fact apply to get more general results on graphical games.

The class of smooth purely atomic games is hence an intrinsically natural category for the study of equilibria existence in game theory. We exhibit this for the existence of Bayesian equilibria in Theorem 4.7, presenting an example of a structure arising with a simple story behind it. We also exhibit this for existence of stationary equilibria in stochastic games in Theorem 5.2. In particular, this result is applied to certain stochastic games arising naturally when one models repeated games with public uncertain information to obtain the existence of equilibria which is stationary in the common beliefs. (As shown in Hellman and Levy (2019), stochastic games arising from this latter model do not, in general, display the required smoothness, but we present a particular sub-class of repeated games that does.) That smooth purely atomic games have an intrinsic natural quality is also exhibited with respect to graphical games in Theorem 8.2. (See Figure 1.) Doubtless,

\footnotetext{
${ }^{4}$ In this case, the 'underlying state space' is the space of players.
} 


\begin{tabular}{|l|l|}
\hline Field & Main Results \\
\hline \hline Bayesian Games & $\begin{array}{l}\text { Measurable Bayesian equilibria exist in } \\
\text { smooth purely atomic games (Theorem 4.7). }\end{array}$ \\
\hline Stochastic Games & $\begin{array}{l}\text { Measurable stationary equilibria exist in } \\
\text { smooth purely atomic games (Theorem 5.2). }\end{array}$ \\
\hline Graphical Games & $\begin{array}{l}\text { Measurable Nash equilibria exist in smooth } \\
\text { purely atomic games (Theorem 8.2). }\end{array}$ \\
\hline Common Priors and Betting & $\begin{array}{l}\text { Measurable acceptable bets exist in smooth } \\
\text { purely atomic games if an acceptable bet ex- } \\
\text { ists on each common knowledge component } \\
\text { (Theorem 9.1). }\end{array}$ \\
\hline
\end{tabular}

FIGURE 1.-Summary of the main results of the paper.

this can be extended to many more types of games; one need only identify the type of game and rely on equilibrium existence in the countable case to obtain the desired result.

\section{PRELIMINARIES}

Claims stated in mathematical formulae, with game-theoretic claims such as equilibrium existence being no exception, can, at their core, be formulated using mathematical logic. Mathematical logic is most often conducted within the framework of first-order logic; see, for example, Enderton (2001). We recall here a very brief and intuitive explanation of the essentials of the finitary and infinitary logics which will be used in the main body of the paper. A much more detailed survey of the topic appears in the Appendix.

\subsection{First-Order Logic}

Mathematical logic is the familiar logic built on a finite base language $\mathcal{L}$ consisting of logical connective symbols (e.g., conjunction, disjunction, and negation); a finite or countable collection of variables; the quantifier symbols $\forall, \exists$; constant symbols; predicate symbols; and function symbols. From the symbols, one may then form terms by repeated composition of function symbols on variables and constant symbols. Atomic formulae are of the form $R\left(t_{1}, \ldots, t_{n}\right)$ for some predicate symbol $R$ (possibly equality) and terms $t_{1}, \ldots, t_{n}$, and finally, well-formed formulae are expressions built from atomic formulae and repeated used of connective and quantifier symbols.

An important distinction is made between free and bound variables. Intuitively, a free variable in a formula represents a variable that, in principle, may be substituted by another term or formula in its place. A bound variable is one that 'has been quantified', that is, if $v$ is a free variable of a formula $\alpha$, then $v$ is bound in the formulae $\forall v \alpha$ and $\exists v \alpha$. A formula with no free variables is called a sentence. (A formal definition of free and bound variables appears in the Appendix.)

\subsection{Infinitary Logic}

In subsequent sections, we will make use of a slight extension of the standard first-order logic, the language of infinitary languages, which we present very briefly here (for more 
on this subject, see the elaboration in the Appendix to this paper or a standard textbook such as Keisler (1971)).

The language of first-order logic can only handle expressions involving a finite number of variables; it is silent with regard to infinite collections of variables. However, dealing with expressions relating to an infinite collection of variables is of direct importance for this paper. To take one example, we will wish to have a formal way of expressing concepts such as 'player $i$ plays a mixed action', or to state the same thing in greater detail, that 'player $i$ places weight $x$ on action $a^{i}$ in state $t$ ', where the number of possible states is countably infinite. Similarly, we may wish to state that the payoff under a mixed action profile in a dynamic programming problem is a particular value, where the number of states that could possibly be visited in the course of process is countably infinite. We will also need a formal way to express convergence, limits, summation, and summability of infinite series, all of which clearly cannot be expressed in standard first-order logic, as formulae in the latter can only accept finitely many variables. Expressing these concepts, especially in a quantifier-free manner as needed for our theorems, is enabled by the use of the language denoted $\mathcal{L}_{\omega_{1}, \omega}$.

Simply put, in this language, in addition to the first-order formulae, we allow formulae of the form $\bigwedge_{n=1}^{\infty} \alpha_{n}$ and $\bigvee_{n=1}^{\infty} \alpha_{n}$, whenever $\left(\alpha_{n}\right)_{n=1}^{\infty}$ is a countable collection of formulae. (If $F_{n}$ is the collection of free variables in $\alpha_{n}$ for each $n$, then $\bigcup_{n} F_{n}$ is the collection of free variables in the formula $\bigwedge_{n=1}^{\infty} \alpha_{n}$ and in the formula $\bigvee_{n=1}^{\infty} \alpha_{n}$.) The intuitive meanings of these countable conjunctions and disjunctions should be clear; as stated above, a more detailed explanation is provided in the Appendix.

For example, in the $\mathcal{L}_{\omega_{1}, \omega}$ language $\mathcal{L}=(+, \cdot,>, 0,1)$, we can state the expression "the sequence $\left(x_{n}\right)_{n=1}^{\infty}$ converges to $L$ " over the real numbers via the formula

$$
\alpha\left(\left(x_{n}\right)_{n=1}^{\infty}, L\right)=\forall \varepsilon\left(\varepsilon>0 \rightarrow \bigvee_{N \in \mathbb{N}} \bigwedge_{n>N}\left(x_{n}-L\right)^{2}<\varepsilon^{2}\right),
$$

where $a^{2}$ is shorthand for $a \cdot a$. If we wish to make an equivalent quantifier-free statement (over $\mathbb{R}$ ), we may write

$$
\alpha\left(\left(x_{n}\right)_{n=1}^{\infty}, L\right)=\bigwedge_{K \in \mathbb{N}} \bigvee_{N \in \mathbb{N}} \bigwedge_{n>N} K^{2} \cdot\left(x_{n}-L\right)^{2}<1 .
$$

\subsection{Countable Borel Equivalence Relations}

A Polish space is a separable and completely metrizable space. Measurability without further qualification in this paper, in the context of a Polish space $X$, will be understood to mean measurability with respect to the Borel $\sigma$-algebra of $X$.

A relation $\mathcal{E}$ on a Polish space $\Omega$ is said to be Borel if the set $\{(x, y) \in \Omega \times \Omega \mid x \mathcal{E} y\}$ is a Borel subset of $\Omega \times \Omega$. An equivalence relation is said to be countable if each equivalence class, referred to as a class or an atom, is countable. We will abbreviate countable Borel equivalence relation as CBER. Similarly, an equivalence relation is finite if each of its equivalence classes is finite. A Borel relation that is a function is a Borel function. ${ }^{6}$ A CBER is said to be aperiodic if each class is infinite.

\footnotetext{
${ }^{5}$ Typically, one writes a logical language by specifying only the symbols, as in $\mathcal{L}=(+, \cdot,>, 0,1)$; the logical connectives are omitted because they are always present, and the set of variables typically do not need to be specified separately.

${ }^{6}$ Continuous functions are Borel but not every Borel function is continuous.
} 
A very central definition from descriptive set theory that is used extensively in this paper is the following:

DEFINITION 2.1: A Borel equivalence relation $\mathcal{E}$ on a Polish space $\Omega$ is smooth if there is a Polish space $Z$ and a Borel function $\psi: \Omega \rightarrow Z$ such that, for all $x, y \in \Omega$,

$$
x \mathcal{E} y \quad \Longleftrightarrow \quad \psi(x)=\psi(y)
$$

(i.e., the classes of $\mathcal{E}$ are precisely the level sets of $\psi$ ).

If $\mathcal{E}$ is the common knowledge relation, a function $\psi$ witnessing the smoothness of the relation can be thought of as an auxiliary tool that enables us to ascertain when $x$ and $y$ are in the same common knowledge component: that occurs if and only if $\psi(x)=\psi(y)$.

EXAMPLE 2.2: On $\Omega=\mathbb{R}^{N}$, the relation given $x \sim_{\mathcal{E}} y$ iff $x-y \in \mathbb{Z}^{N}$ is smooth, as, if we define $\psi: \mathbb{R}^{N} \rightarrow[0,1)^{N}$ by

$$
\psi\left(x_{1}, \ldots, x_{N}\right)=\left(x_{1}-\left\lfloor x_{1}\right\rfloor, \ldots, x_{N}-\left\lfloor x_{N}\right\rfloor\right),
$$

where $\lfloor a\rfloor=\max \{k \in \mathbb{Z} \mid k \geq a\}$ denotes the integer part of $a$, then $\psi(x)=\psi(y)$ if and only if $x \sim_{\mathcal{E}} y$.

EXAMPLE 2.3: On the unit circle $S^{1}=\{z \in \mathbb{C}|| z \mid=1\}$, observe an irrational rotation $T: S^{1} \rightarrow S^{1}$ given by $T(z)=z \cdot e^{2 \pi i \alpha}$ for fixed irrational $\alpha \in \mathbb{R}$. It is well known that if $\psi: S^{1} \rightarrow Z$ is Borel for some Borel space $Z$, such that $\psi=\psi \circ T$, then $\psi$ is constant.

A transversal of $\mathcal{E}$ is a set $T \subseteq X$ which intersects each $\mathcal{E}$ equivalence class at exactly one point. It is easy to see that if a Borel $\mathcal{E}$ has a Borel transversal, then it is smooth: intuitively, the map $\psi(x)=$ 'the only element of $T$ that is $\mathcal{E}$-equivalent to $x$ ' witnesses the smoothness of $\mathcal{E}$. For CBER's, the converse is true as well (see Proposition 2.5).

EXAMPLE 2.4: In Example 2.2, the set $[0,1)^{N}$ is clearly a Borel transversal.

From this, one can show that if every equivalence class of $\mathcal{E}$ is finite, then $\mathcal{E}$ is smooth. ${ }^{7}$ However, for CBER's, which are the focus of much of the material of this paper, matters are not as simple.

When $\mathcal{E}$ is an equivalence relation, for each $x \in \Omega$ denote the equivalence class containing $x$ by $[x]_{\mathcal{E}}$. A set $B \subseteq \Omega$ is said to be saturated with respect to $\mathcal{E}$ if it is the union of $\mathcal{E}$-equivalence classes, that is, if there is a set $A \subseteq \Omega$ such that $B=[A]_{\mathcal{E}}:=\bigcup_{x \in A}[x]_{\mathcal{E}}$. The collection of all the Borel $\mathcal{E}$-saturated sets of a Borel equivalence relation $\mathcal{E}$ forms a $\sigma$-algebra, denoted $\sigma(\mathcal{E})$.

Given a Polish state space $\Omega$ and a $\operatorname{CBER} \mathcal{E}$, we let $\Omega / \mathcal{E}$ denote the quotient space whose elements are the equivalence classes by $\mathcal{E}$, and the induced $\sigma$-algebra consists of

${ }^{7}$ Consider the set

$$
T=\{x \in X \mid \forall y \in X, x \mathcal{E} y \Longrightarrow x \leq y\},
$$

that is, the set of the $\leq$-elements of the $\mathcal{E}$ equivalence classes, for any Borel linear order on the domain of $\mathcal{E}$; such exists by a theorem of Kuratowski (see Section 15.B of Kechris (1995)). This $T$ is seen to be Borel and a transversal of $\mathcal{E}$, hence finiteness of the $\mathcal{E}$-classes is sufficient for smoothness (see, e.g., Example 6.1 of Kechris and Miller (2004)). 
precisely the images of the $\mathcal{E}$-saturated Borel sets in $\Omega$ under the quotient map $\iota: \Omega \rightarrow$ $\Omega / \mathcal{E}$; this is the finest $\sigma$-algebra on $\Omega / \mathcal{E}$ such that $\iota$ is measurable.

We will make repeated use of the following proposition, which follows from Propositions 6.3 and 6.4 of Kechris and Miller (2004) and the discussion preceding them.

PROPOSITION 2.5: The following conditions are equivalent for a CBER $\mathcal{E}$ on a Polish space $\Omega$ :

(a) $\mathcal{E}$ is smooth.

(b) There is a Borel transversal for $\mathcal{E}$.

(c) There is a Borel set intersecting each class of $\mathcal{E}$ in a finite non-empty set.

(d) The quotient space $\Omega / \mathcal{E}$ is standard Borel. ${ }^{8}$

The following is a slight variation of the Lusin-Novikov theorem; for example, Kechris (1995, Theorem 18.10); see also Dougherty, Jackson, and Kechris (1994, Theorem 5.1).

PROPOSITION 2.6: Let $\mathcal{E}$ be a smooth $C B E R$ on a Polish space $\Omega$, and let $S$ be a countably infinite set. Then there is a Borel mapping $\Phi: \Omega \rightarrow S$ such that, for each $\mathcal{E}$-class $C$ of $\Omega$, the restriction $\left.\Phi\right|_{C}: C \rightarrow S$ is injective, and if $C$ is infinite, then $\Phi(C)=S$. If $S=\left\{s_{1}, s_{2}, s_{3}, \ldots\right\}$ is an enumeration of $S$, then $\Phi$ can be chosen such that $\Phi(C)=\left\{s_{1}, s_{2}, \ldots, s_{n}\right\}$ when $C$ is of cardinality $n$.

In words, Proposition 2.6 states that for a smooth CBER over $\Omega$ and a given countably infinite set $S$, it is possible to define a mapping $\Phi: \Omega \rightarrow S$ in a Borel manner such that simultaneously each and every equivalence class $C \subseteq \Omega$ of the CBER $\mathcal{E}$ under is mapped injectively to $S$ by $\Phi$. This will play a key role in several results.

Recall that a subset of a Polish space $X$ is analytic (a.k.a., Souslin) if it is the image of a Borel function $f: Z \rightarrow X$ from another Polish space $Z$. A function $g: X \rightarrow Z$ between Polish spaces is analytically measurable if, for each Borel $B \subseteq Z, g^{-1}(B)$ is in the $\sigma$-algebra generated by the analytic sets. In that case, if $\mu$ is a Borel probability measure on $X$, then there is a Borel set $\hat{X} \subseteq X$ of full measure such that the restriction $\left.g\right|_{\hat{X}}: \hat{X} \rightarrow Z$ is Borel (this follows from, e.g., Kechris (1995, Theorem 21.10)).

\section{A SELECTION THEOREM}

The proofs of several theorems in this paper will follow the following general paradigm: they will relate to some game over an uncountable state space such that this state space can be considered to be composed of disjoint countable sets, each of which is 'naturally' identified as the equivalence class of a Borel equivalence relation, and furthermore, each such countable Borel equivalence class can be identified with at least one measurable equilibrium (as appropriately defined for the type of game at hand).

The goal will then be to show that (assuming that the Borel equivalence relation is smooth) a measurable equilibrium over the entire uncountable state space exists by the construction of a formula that 'witnesses' the existence of an equilibrium over the countable Borel equivalence classes. The selection theorem of this section is the key element to achieving that.

\footnotetext{
${ }^{8}$ That is, there is a Borel-measurable bijection between it and a Polish space.
} 


\subsection{Main Selection Theorem}

We work with the following:

- A fixed Polish space $\Omega$ with a CBER $\mathcal{E}$, and a Polish space $X$. $\Omega$ will be the state space, and $X$ will be a parameter space. Our examples will almost all ${ }^{9}$ suppose that $X=\mathbb{R}$, but the theory in full generality allows any Polish $X$.

- A finite or countably infinite set $S$ such that the equivalence classes of $\mathcal{E}$ are each of cardinality $S$.

- A tuple $\bar{F}_{D}=\left(N_{1}, \ldots, N_{K}\right)$ with $N_{j} \in \mathbb{N}$ (where possibly $K=\infty$ ), and a corresponding sequence of Borel functions $\zeta_{1}, \ldots, \zeta_{K}$ with $\zeta_{j}: \Omega^{N_{j}} \rightarrow X$ for each $j$.

- Denote $F_{D}=\sum_{j} N_{j}$ (which may be infinite).

- Let $F_{U} \in \mathbb{N} \cup\{0, \infty\}$.

For a given $\mathcal{E}$-equivalence class $C \subseteq \Omega$ and a given bijection $\phi: C \rightarrow S$, define a function $\zeta[\phi]: \prod_{j} S^{N_{j}} \rightarrow X^{K}$ as the function whose $j$ th component is $\zeta_{j}\left(\phi^{-1}\left(s_{1}\right), \ldots\right.$, $\left.\phi^{-1}\left(s_{N_{j}}\right)\right)$.

In other words, for each $1 \leq j \leq K$, take the corresponding element $N_{j}$ of $\bar{F}_{D}=$ $\left(N_{1}, \ldots, N_{K}\right)$, and map each sequence $\left(s_{1}, \ldots, s_{N_{j}}\right) \in S^{N_{j}}$ to $X$ via $\zeta_{j}\left(\phi^{-1}\left(s_{1}\right), \ldots\right.$, $\phi^{-1}\left(s_{N_{j}}\right)$ ). Formally,

$$
\zeta[\phi]:=\left(\zeta_{j}\left(\phi^{-1}\left(s_{1}\right), \ldots, \phi^{-1}\left(s_{N_{j}}\right)\right) \mid 1 \leq j \leq K, s_{1}, \ldots, s_{N_{j}} \in S\right) .
$$

The theorem here involves structures $X^{S \times F_{D}}$, which we will call the determined variables, and $X^{S \times F_{U}}$, which are the undetermined variables; this terminology will be further clarified in Section 3.2. One may think of the determined variables as corresponding to the data given in the underlying situation while the undetermined variables correspond to what we wish to construct.

To keep a running example in mind, the reader may consider Bayesian games (to be discussed in full detail in Section 4.3). In a Bayesian game, the determined variables are the collection of the beliefs of each player regarding the probabilities of the states along with the collection of the payoffs granted to each player contingent on the state and the profile of selected actions; these are the given data of the underlying situation. The undetermined variables are the strategies that the players may choose. The equivalence classes are the common knowledge components of the game. The crux of the matter is to show that if the equivalence relation is smooth and for each equivalence class (within the game data) we can associate certain strategies, then there is a Borel-measurable way to construct our desired equilibrium strategy over the entire game data.

THEOREM 3.1: Let $\mathcal{E}$ be smooth and let a set $Z$ satisfying

$$
Z \subseteq X^{S \times\left(F_{D} \cup F_{U}\right)}
$$

be Borel. Furthermore, for each equivalence class $C \in \mathcal{E}$ and $\phi: C \rightarrow S$, define

$$
Z[\zeta, \phi]:=\left\{x^{F_{U}} \mid\left(\zeta[\phi], x^{F_{U}}\right) \in Z\right\} .
$$

(Intuitively, $Z[\zeta, \phi]$ is the collection of $S \times F_{U}$-tuples of undetermined variables which complete the $F_{D}$-tuple $\zeta[\phi]$ of determined variables to an element of $Z$.)

\footnotetext{
${ }^{9}$ In the proof of Theorem 7.1, we will allow $X$ to be a general compact metric space.
} 


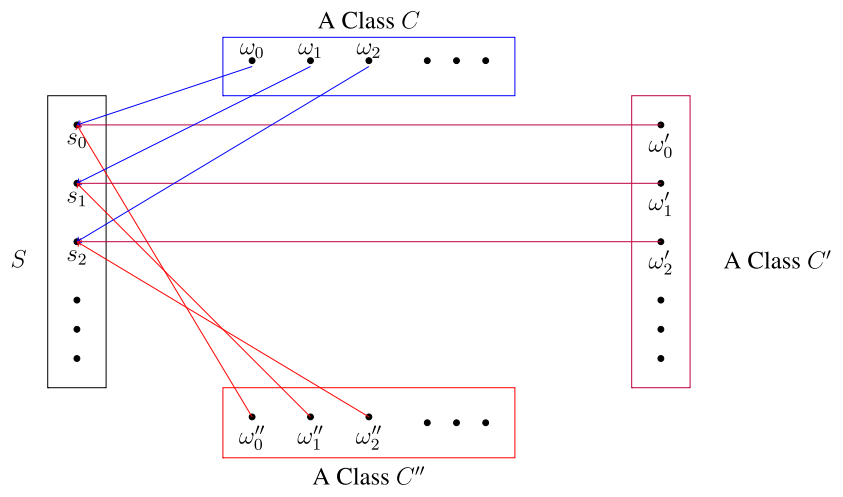

FIGURE 2.-The mapping $\Phi$.

(a) Suppose that for each equivalence class $C$ of $\mathcal{E}$, there is a bijection $\phi: C \rightarrow S$ such that $Z[\zeta, \phi] \neq \emptyset$. Then there exist analytically measurable mappings $\Phi: \Omega \rightarrow S$ and $\Psi: \Omega / \mathcal{E} \rightarrow$ $X^{F_{U}}$ such that for each equivalence class $C$ of $\mathcal{E}$ :

- The restriction $\left.\Phi\right|_{C}: C \rightarrow S$ is bijective.

- $\Psi(C) \in Z\left[\zeta,\left.\Phi\right|_{C}\right]$, that is, $\left(\zeta\left[\left.\Phi\right|_{C}\right], \Psi(C)\right) \in Z$.

(b) If, for each equivalence class $C$ of $\mathcal{E}$, it is the case that for any bijection $\phi: C \rightarrow S$, $Z[\zeta, \phi]$ is non-empty and $\sigma$-compact, then $\Phi$ and $\Psi$ can be chosen to be Borel-measurable.

See Figure 2 for an example of such a mapping $\Phi$.

We sketch the proof here (the full proof is in the Appendix). Typical measurable selection theorems select measurable functions $f: \Theta \rightarrow \Lambda$ from a collection of measurable (in an appropriate sense) correspondences $F: \Theta \rightarrow \Lambda$. The difficulty in applying such theorems directly to our setup is that for each component $C$ of $\mathcal{E}$, the collection of desired objects are mappings $F_{U} \times C \rightarrow X$. Going back to the example we are keeping in mind, if the desired objects are Bayesian equilibria over the common knowledge component, then these are mappings from $C \times F_{U} \rightarrow \mathbb{R}$, where $F_{U}$ is the collection of all pure actions, which specify in each state $C$, for each player and each of his pure actions, how much weight to place on that action. Since the space $F_{U} \times C$ varies from component to component, there is no fixed domain of correspondence on which to conduct a straightforward application of a typically styled selection theorem.

By translating from each $C$ to $S$ via such a mapping $\Phi$ (in part (b), this can be done in any arbitrary way that Proposition 2.6 allows), we instead have that for each component $C$, the set of desired objects are mappings on $S \times F_{U}$ to $X$, that is, elements of $X^{S \times F_{U}}$. Now, working with this constant domain, we can appeal to measurable selection theorems. Part (a) is somewhat more intricate, as the mapping $\Phi$ needs to be chosen as well. For this, we first choose any mapping $\hat{\Phi}$ as provided by Proposition 2.6, and then observe that for any other such map $\Phi$ and each fixed component $C,\left.\Phi\right|_{C}$ is a permutation of $\left.\hat{\Phi}\right|_{C}$. We then simultaneously have to choose, in addition to the desired object (e.g., equilibrium), an appropriate permutation to give an appropriate mapping $\Phi$.

REMARK 3.2: In the second part of the above theorem, even if we (only) assume $Z[\zeta, \phi]$ is non-empty for each class $C$ of $\mathcal{E}$ for any bijection $\phi: C \rightarrow S$, but not neces- 
sarily $\sigma$-compact, then there may not be any Borel-measurable selectors. ${ }^{10}$ Similarly, if $Z[\zeta, \phi]$ is non-empty and compact for some $\phi$ but is either empty or non- $\sigma$-compact for others, we may not be able to deduce Borel measurability.

REMARK 3.3: As in Example 3.8 later, Theorem 3.1 can be applied when $F_{U}=0$. (In this case, the mapping $\Psi$ is degenerate, and only the map $\Phi$ is relevant.) Formally, in that case, if $\zeta[\phi] \in Z$, then $Z[\zeta, \phi]=0^{X} \neq \emptyset$, while if $\zeta[\phi] \notin Z$, then $Z[\zeta, \phi]=\emptyset$.

It is useful to pair the selection theorem with the following, Proposition 3.4, whose proof appears here in a footnote. ${ }^{11}$

Proposition 3.4: Given a Polish space $\Omega$ and a CBER $\mathcal{E}$ on $\Omega$, there exists a partition $\Omega_{1}, \Omega_{2}, \ldots, \Omega_{\infty}$ of $\Omega$, consisting of Borel $\mathcal{E}$-saturated sets, such that for each $k=$ $1,2, \ldots, \infty$, each equivalence class of $\mathcal{E}$ in $\Omega_{k}$ is of cardinality $k$.

Since the sets $\Omega_{1}, \Omega_{2}, \ldots, \Omega_{\infty}$ are Borel subsets of a Polish space, they are standard Borel, that is, they themselves are Polish (in some topology inducing their Borel structures); see, for example, Kechris (1995, Corollary 13.4). Note that Theorem 3.1 relies only on the Borel structure of $\Omega$ being standard Borel, not on the specific topology. Hence, it is possible to apply Theorem 3.1, either part (a) or (b), on each partition element simultaneously, even though the classes may be of different cardinalities; by an abuse of notation, we refer to the gluing of all such mappings as $(\Phi, \Psi)$ (and given an enumeration of $S=\left\{s_{1}, s_{2}, \ldots\right\}$, we may assume, as in Proposition 2.6, that when a class $C$ has cardinality $n$, then $\left.\Phi(C)=\left\{s_{1}, \ldots, s_{n}\right\}\right)$.

EXAMPLE 3.5: Fix an integer $N$. Suppose we have an aperiodic ${ }^{12}$ CBER $\mathcal{E}$ on a Borel space $\Omega$, and a Borel function $\zeta: \Omega \rightarrow \mathbb{R}$. We wish to construct a Borel set $B \subseteq \Omega$ such that for each equivalence class $C$ of $\mathcal{E}, B \cap C$ is of size $N$ and $\zeta$ takes different values on each element of $B \cap C$. (Assume $\zeta$ takes at least $N$ different values in each class.) In other words, within each equivalence class of $\mathcal{E}$ we seek to identify a subset of size exactly $N$ in such a way that each element within that subset is assigned a different number in $\mathbb{R}$ via $\zeta$, and to do all this in a Borel manner. There are various ways to go about this, and one way is via the use of Theorem 3.1.

First, we need to define $S, X, K, \bar{F}_{D}=\left(N_{1}, \ldots N_{K}\right)$, and $F_{U}$ as in the discussion prior to Theorem 3.1. Intuitively, the determined element is the function $\zeta$, and what is undetermined is the set of size $N$ that is to be associated with each equivalence class. Let $S$ be

\footnotetext{
${ }^{10}$ This is true even when $\mathcal{E}=i d$, and even when $Z$ is induced by a formula $\alpha$ as in Section 3.2 which is quantifier-free. Take, for example, $\Omega=\mathbb{R}$ and $\mathcal{L}=(+, \cdot,>, 0,1)$. Every open set can be written as a countable union of intervals with rational end-points, hence every closed set can be defined in $\mathcal{L}_{\omega_{1}, \omega}$. However, it is known that not every correspondence with a closed graph (and non-empty values) between Polish spaces need possess a Borel-measurable selection; see, for example, Kechris (1995, Example 14.3).

${ }^{11}$ Proof of Proposition 3.4: The Feldman-Moore theorem (Feldman and Moore (1975)) states that every CBER is induced by a countable group of Borel actions, $G$, which we enumerate $G=\left\{g_{1}, g_{2}, \ldots\right\}$. Define $\psi: \Omega \rightarrow \mathbb{N} \cup\{\infty\}$ by

$$
\psi(\omega)=\sum_{n=1}^{\infty} \prod_{k<n} \zeta\left(g_{k}(\omega), g_{n}(\omega)\right),
$$

where $\zeta: \Omega \rightarrow\{0,1\}$ is 1 if and only if the coordinates are different. (The empty product is 1.$) \psi(\omega)$ is then clearly the cardinality of the $\mathcal{E}$-class of $\omega$.

${ }^{12}$ Recall that a CBER is aperiodic if each one of its equivalence classes is infinite.
} 
an arbitrary set of the cardinality of the equivalence classes, that is, countably infinite; let $X=\mathbb{R}, K=1$ (hence the tuple $\bar{F}_{D}=\left(N_{1}, \ldots N_{K}\right)$ collapses to the singleton $N_{1}=F_{D}=1$ ), and then $\zeta_{1}: \Omega^{N_{1}} \rightarrow X$ is simply the given 'input data' function $\zeta: \Omega \rightarrow \mathbb{R}$. Let $F_{U}=1$. For any equivalence class $C$ of $\mathcal{E}$ and mapping $\phi: C \rightarrow S$ that is a bijection, $\zeta[\phi]$ maps $S$ to $\mathbb{R}$ by $\zeta[\phi](s)=\zeta\left(\phi^{-1}(s)\right)$.

We can identify each subset of $S$ with an element of $\{0,1\}^{S} \subseteq \mathbb{R}^{S}$. Take $Z$ to be

$$
\begin{aligned}
Z= & \left\{(x, y) \mid x \in \mathbb{R}^{S}, y \in\{0,1\}^{S} \text { such that the set corresponding to } y\right. \text { is } \\
& \text { of size } \left.N \text {, and if } s_{1} \neq s_{2} \in S \text { but } y\left(s_{1}\right)=y\left(s_{2}\right)=1 \text {, then } x\left(s_{1}\right) \neq x\left(s_{2}\right)\right\} .
\end{aligned}
$$

$Z$ can be shown to be Borel. ${ }^{13}$ We have thus formalized our initial intuition: $\zeta[\phi] \in \mathbb{R}^{S}$ represents the determined variables, and $\{0,1\}^{S}$ the undetermined variables.

For an equivalence class $C$ of $\mathcal{E}$ and a bijection $\phi: C \rightarrow S, Z[\zeta, \phi]$ is given by (3.3), and consists of those elements $y \in\{0,1\}^{S}$ such that $(\zeta[\phi], y) \in Z$. To apply Theorem 3.1, note that we have supposed that $Z[\zeta, \phi]$ is non-empty for each class $C$ of $\mathcal{E}$ and each bijection $\phi: C \rightarrow S$, as we have assumed that $\zeta$ takes on at least $N$ different values in each class. Then by the result of the theorem, there exist $\Phi: \Omega \rightarrow S$ and $\Psi: \Omega / \mathcal{E} \rightarrow \mathbb{R}$ such that, for each equivalence class $C$ of $\mathcal{E},\left.\Phi\right|_{C}: C \rightarrow S$ is bijective, and $\left(\zeta\left[\left.\Phi\right|_{C}\right], \Psi(C)\right) \in Z$. This formally states what we want: $\Phi$ bijectively identifies $C$ with $S$, and then $\left(\zeta\left[\left.\Phi\right|_{C}\right], \Psi(C)\right) \in$ $Z$ can be translated to saying we have identified a subset of $C$ of size $N$ satisfying the condition that distinct elements of that subset are mapped to distinct real numbers via $\zeta$.

Finally, using the notation $\iota: \Omega \rightarrow \Omega / \mathcal{E}$ for the quotient mapping, define $B \subseteq \Omega$ by

$$
B:=\{\omega \in \Omega \mid \Psi(\iota(\omega))(\Phi(\omega))=1\} .
$$

$B \in \Omega$ is the set we sought from the start, and by construction it is Borel.

\subsection{Correspondences Induced by Formulae}

In this section, we add to the approach of Theorem 3.1 an explicit relation to logical formulae. More specifically, we seek to use that theorem to obtain Borel relations that can be defined by satisfaction of a formula, which is the content of Proposition 3.6.

We continue with the same notation as in Section 3.2, namely, a fixed Polish space $\Omega$ with CBER $\mathcal{E}$, a Polish space of parameters $X$ (usually $\mathbb{R}$ ), a fixed countable set $S$, Borel functions $\zeta_{j}: \Omega^{N_{j}} \rightarrow X$ for $j=1, \ldots, K$, and $\bar{F}_{D}=\left(N_{1}, \ldots, N_{K}\right)$. We also assume here that we have the following:

- A language $\mathcal{L}$. We note that $\mathcal{L}$ may include variables which, for convenience, are indexed by $S$, or $S \times S$, etc, that is, collections of variables $\left(x_{s}\right)_{s \in S},\left(y_{s, t}\right)_{s, t \in S}$, etc; we may denote these $(x(s))_{s \in S},(y(s, t))_{s, t \in S}$ to avoid excessive subscripts.

- For every $n$, for each $n$-place predicate symbol $R \in \mathcal{R}$, let $R_{X} \subseteq X^{n}$ be a Borel relation, and for each $n$-place function symbol (including possibly constant symbols) $f \in \mathcal{F}$, let $f_{X}: X^{n} \rightarrow X$ be a Borel function. (In this way, by construction, the universe $X$, together with the assignments $R_{X}$ for each relation $R$ and $f_{X}$ for each function $f$ in the language, forms a model of $\mathcal{L}$.) Relations such as $=, \leq,<$ and functions such as,,$+- \cdot$ will always be defined as relations $\subseteq X \times X$ and functions $X \times X \rightarrow X$ in the natural way.

\footnotetext{
${ }^{13}$ We will return to this in Section 3.3, using the machinery of Section 3.2.
} 
- In addition to $\bar{F}_{D}$, a tuple of $\bar{F}_{U}=\left(M_{1}, \ldots, M_{L}\right), M_{j} \in \mathbb{N}$ for each $j=1, \ldots, L$ (we may have $\left.{ }^{14} L=0, \infty\right)$, denoting the dimensions for the undetermined variables, as elaborated below; denote $F_{U}=\sum_{j} M_{j}$.

- Let $\alpha$ be a formula in $\mathcal{L}_{\omega_{1}, \omega}$. Assume that $\alpha$ 's free variables can be partitioned into two sets:

$-\left(x_{j}\left(s_{1}, \ldots, s_{N_{j}}\right)\right)$ for each $j=1, \ldots, K$ and each $s_{1}, \ldots, s_{N_{j}} \in S$, where recall $\bar{F}_{D}=$ $\left(N_{1}, \ldots, N_{K}\right)$ are such that $\zeta: \Omega^{N_{j}} \rightarrow X$. These are the determined $S$-variables.

$-\left(x_{j}\left(t_{1}, \ldots, t_{M_{j}}\right)\right)$ for each $j=1, \ldots, L$ and each $t_{1}, \ldots, t_{M_{j}} \in S$, where recall $\bar{F}_{U}=$ $\left(M_{1}, \ldots, M_{L}\right)$. These are the undetermined $S$-variables.

In this case, we wish to apply Theorem 3.1 to the set:

$$
Z=\left\{x \in X^{S \times\left(F_{D} \times F_{U}\right)} \mid \alpha(x) \text { holds }\right\} .
$$

Returning again to the specific example of Bayesian games, as mentioned before, the determined variables are the structure of the game itself and the undetermined variables are strategy profiles chosen by the players. To express the semantics that we wish to ascribe to the relations between these variables, we may use a logical formula $\alpha$ whose free variables are payoffs, beliefs, and strategies, w.r.t. the state space $S$, that is, we wish to express that beliefs are constant on a player's belief components, that probability weights are between zero and one, etc. Furthermore, we wish to identify not just any strategy profiles but those that form equilibria. To specify all this, we use infinitary logical formulae to formulate what we require the undetermined variables to satisfy.

The following proposition follows by induction on the construction of $\alpha$ :

Proposition 3.6: Let the set $Z$ of Equation (3.2) be defined by logical satisfaction as $Z=\left\{x \in X^{S \times\left(F_{D} \times F_{U}\right)} \mid \alpha(x)\right.$ holds $\}$ for a formula $\alpha \in \mathcal{L}_{\omega_{1}, \omega}$. Then $Z$ is Borel if $\alpha$ is quantifierfree. ${ }^{15}$

\subsection{More Examples}

We expand on a previous example, and present an additional one.

EXAMPLE 3.7: Returning to Example 3.5, recall that the set $Z$ to which we applied Theorem 3.1 was defined in Equation (3.4), without any reference to a logical formula. Let, then, $\tilde{S}^{N}$ stand for the collection of $N$-tuples of distinct elements of $S$ (this is countable), in the language $\mathcal{L}=(0,1, \cdot)$, and consider the following formula $\alpha$ :

$$
\begin{gathered}
\alpha\left((x(s))_{t \in S},(y(s))_{s \in S}\right)=\bigwedge_{s \in S}(y(s)=0 \vee y(s)=1) \\
\bigwedge_{\substack{\left(s_{1}, \ldots, s_{N}\right) \in \tilde{S}^{N}\\
}}\left(\bigwedge_{j=1}^{N} y\left(s_{j}\right)=1 \bigwedge_{s \neq s_{1}, \ldots, s_{N}} y(s)=0\right) \\
\bigwedge_{(s, t) \in \tilde{S}^{2}}(x(s)=x(t) \rightarrow y(s) \cdot y(t)=0),
\end{gathered}
$$

\footnotetext{
${ }^{14}$ For an example with $L=0$, see Example 3.8 of Section 3.3.

${ }^{15} \mathrm{~A}$ formula is quantifier-free if it can be constructed without use of the universal quantifier $\forall$ or the existential quantifier $\exists$.
} 
where the first part states that $y \in\{0,1\}^{S}$, the second states that $y(s)=1$ for precisely $N$ values of $S$, and the third states that if $x(s)=x(t)$, then either $y(t)=0$ or $y(s)=0$. In this case, in the notation of Section 3.2, $\bar{F}_{U}=(1), F_{U}=1$.

Then $Z$ can be written as

$$
Z=\left\{(x, y) \mid x \in \mathbb{R}^{S \times S}, y \in \mathbb{R}^{S}, \text { and } \alpha(x, y) \text { holds }\right\}
$$

and we may directly apply Proposition 3.6. (Note that $\alpha(x, y)$ holding implies $y \in\{0,1\}^{S}$.)

EXAMPLE 3.8: Let $\mathcal{E}$ be a smooth aperiodic CBER on a Polish space $\Omega$, and let $\zeta: \Omega \rightarrow$ $\mathbb{R}$ be Borel. Take $S=\mathbb{Z}$. We wish to obtain an analytically measurable $\Phi: \Omega \rightarrow \mathbb{Z}$, bijective when restricted to each equivalence class, such that:

- If $C$ is an equivalence class such that the sequence $(\zeta(\omega)))_{\omega \in C}$ has no maximum, no minimum, and no infinitely repeated elements, then $k<n$ implies $\zeta\left(\left.\Phi\right|_{C} ^{-1}(k)\right)<$ $\zeta\left(\left.\Phi\right|_{C} ^{-1}(n)\right)$.

- Otherwise, $\left.\Phi\right|_{C}$ is an arbitrary bijection.

To accomplish this, define, in the language $\mathcal{L}=(>,=)$,

$$
\beta\left(\left(x_{z}\right)_{z \in \mathbb{Z}}\right)=\bigwedge_{k \in \mathbb{Z}}\left[\left(\bigvee_{l \in \mathbb{Z}}\left(x_{l}>x_{k}\right)\right) \vee\left(\bigvee_{m \in \mathbb{Z}}\left(x_{k}>x_{m}\right)\right)\right],
$$

that is, there is no maximal or minimal element in $\left(x_{z}\right)_{z \in \mathbb{Z}}$, and,

$$
\gamma\left(\left(x_{z}\right)_{z \in \mathbb{Z}}\right)=\bigvee_{k \in \mathbb{Z}} \neg\left[\bigwedge_{n \in \mathbb{N}|m|>n} \bigvee_{m}\left(x_{m}=x_{k}\right)\right],
$$

that is, for each sequence element, there are only finitely many others equal to it. Finally, define

$$
\alpha\left(\left(x_{z}\right)_{z \in \mathbb{Z}}\right)=\left(\beta\left(\left(x_{z}\right)_{z \in \mathbb{Z}}\right) \wedge \gamma\left(\left(x_{z}\right)_{z \in \mathbb{Z}}\right)\right) \rightarrow \bigwedge_{k, m \in \mathbb{Z}, k>m}\left(x_{k}>x_{m}\right) .
$$

Here, $\alpha$ has no undetermined variables, that is, $F_{U}=0$, and $\bar{F}_{D}=(1)$ in the notation of Section 3.2. Putting it all together, we satisfy what is described in Remark 3.3. Note that for each equivalence class $C$ and bijection $\phi: C \rightarrow \mathbb{Z}$, if $\zeta[\phi]:=\left(\zeta\left(\phi^{-1}(k)\right)_{k \in \mathbb{Z}}\right.$ satisfies $\beta \wedge \gamma$, then $\zeta[\tilde{\phi}]=$ satisfies $\beta \wedge \gamma$ for any other bijection $\tilde{\phi}: C \rightarrow \mathbb{Z}$; the same is not true of $\alpha$ as a whole. It is clear, however, that if $\zeta[\phi]$ satisfies $\beta \wedge \gamma$ for some $\phi$, then there exists a $\phi^{\prime}$ such that $\zeta\left[\phi^{\prime}\right]$ satisfies $\alpha$ as well; if $x$ does not satisfy $\beta \wedge \gamma$, then for all bijections $\tilde{\phi}: C \rightarrow \mathbb{Z}, \zeta[\tilde{\phi}]$ satisfies $\alpha$.

Either way, for each equivalence class $C$, some bijection $\phi: C \rightarrow \mathbb{Z}$ ensures that $\zeta[\phi]$ satisfies $\alpha$. Hence, applying the first part of Theorem 3.1, using Equation (3.5), we conclude that $\Phi: \Omega \rightarrow \mathbb{Z}$ is a bijection, such that $\left.\phi\right|_{C}$ is a bijection for each equivalence class $C \in \Omega / \mathcal{E}$, and in addition, if $x:=\zeta\left[\left.\Phi\right|_{C}\right]$ satisfies $\beta \wedge \gamma$, that is, has no maximum/minimum and no infinitely repeated number, then it is also the case that $\cdots>x_{2}>x_{1}>x_{0}>x_{-1}>$ $\cdots$. This achieves what we set out to attain.

\section{BAYESIAN GAMES WITH PURELY ATOMIC KNOWLEDGE SPACES}

\subsection{Knowledge Spaces}

Let $I$ be a non-empty, finite set of players and $\Omega$ a Polish space of states. With each player $i \in I$ we associate a Borel equivalence relation over $\Omega$ denoted $\mathcal{E}^{i}$, called $i$ 's $k n o w l$ - 
edge relation. ${ }^{16}$ Adopting the convention that $\mathscr{E}$ stands for the profile of knowledge relations $\left(\mathcal{E}^{i}\right)_{i \in I}$, a knowledge space is then a triple $(\Omega, I, \mathscr{E})$. Given a knowledge space $(\Omega, I, \mathscr{E})$, the equivalence relation induced by $\mathscr{E}$, which will be denoted by $\mathcal{E}$ and known as the common knowledge relation, is the transitive closure of the union $\bigcup_{i \in I} \mathcal{E}^{i}$, that is, the smallest equivalence relation containing each element in $\mathscr{E} .{ }^{17}$

\subsection{Purely Atomic Knowledge and Type Spaces}

DEFINITION 4.1: A knowledge space such that, for all $i \in I$, each equivalence class of $\mathcal{E}^{i}$ is finite or countably infinite will be called a purely atomic knowledge space.

Purely atomic knowledge spaces were studied, as mentioned, in multiple works, for example, Simon (2000, 2003), Hellman (2014b), Hellman and Levy (2017), Lehrer and Samet (2011).

Fix a knowledge space $(\Omega, I, \mathscr{E})$. For each $i \in I$, a type function $t^{i}$ is a mapping $t^{i}: \Omega \rightarrow$ $\Delta(\Omega)$ that is $\sigma\left(\mathcal{E}^{i}\right)$-measurable ${ }^{18}$ and satisfies $t_{\omega}^{i}(A)=1$ whenever $\omega \in A \in \sigma\left(\mathcal{E}^{i}\right)$.

Adopting the convention that $t$ stands for the tuple $\left(t^{i}\right)_{i \in I}$, a triple $(\Omega, I, t)$ is called a type space. A type space implicitly defines the knowledge relations $\mathcal{E}^{i}$ underlying the type functions: $\omega \mathcal{E}^{i} \omega^{\prime}$ (i.e., $\left.\left(\omega, \omega^{\prime}\right) \in \mathcal{E}^{i}\right)$ if and only if $t_{\omega}^{i}=t_{\omega^{\prime}}^{i}$. Intuitively, $t_{\omega}^{i}(B)$ is the probability player $i$ associates to the set $B$ in state $\omega$.

DEFINITION 4.2: A type space such that, for all $i \in I$ and all $\omega \in \Omega$, the type $t_{\omega}^{i}$ is purely atomic will be called a purely atomic type space.

Clearly, any type space on a purely atomic knowledge space is also purely atomic. We will henceforth always assume that knowledge spaces (and, hence, type spaces) are purely atomic.

DEFINITION 4.3: A type space such that $t_{\omega}^{i}[\omega]>0$ for all $i \in I$ and all $\omega \in \Omega$ is called positive.

If types are purely atomic and positive, then the knowledge space is purely atomic. If positivity is not assumed, then types may be purely atomic but not the knowledge space.

The next concept, that of smoothness, is central to the results of this paper.

DEFINITION 4.4: A purely atomic type space is smooth if the state space $\Omega$ along with the common knowledge equivalence relation $\mathcal{E}$ is smooth.

\footnotetext{
${ }^{16}$ Intuitively, the unions of classes of $\mathcal{E}^{i}$ represent the events that player $i$ can identify; hence, $\sigma\left(\mathcal{E}^{i}\right)$ is the set of Borel events that player $i$ can identify. Most game-theory models work with partitionally generated type spaces. In such models, where $\Omega$ is finite or countable, each player $i$ has a partition $\Pi^{i}$ of $\Omega$. This approach suffers from a difficulty in the case of a continuum of states, since the partition has to 'agree' with the measurable structure. In addition, in the continuum case, one cannot work with arbitrary unions of partitions elements; only Borel unions are admissible. Our approach differs from the more classical approach given in Nielsen (1984) and Brandenburger and Dekel (1987) in favor of defining knowledge via relations (instead of $\sigma$-algebras), which is better suited for the class of purely atomic types that will concern us. Our approach also differs from the 'types' approach of Milgrom and Weber (1985). See the discussion in Hellman and Levy (2017) for a comparison.

${ }^{17}$ In terms that may be more familiar, $\mathcal{E}$ is the common knowledge equivalence relation. The class of the common knowledge relation $\mathcal{E}$ containing $\omega$ is called the common knowledge component containing $\omega$, and is denoted $\mathscr{C}(\omega)$.

${ }^{18}$ Meaning that for Borel $A \subseteq \Omega, \omega \rightarrow t^{i}(A \mid \omega)$ is $\sigma\left(\mathcal{E}^{i}\right)$-measurable.
} 
Section A presents both a sufficient condition for the smoothness of a type space; the next section presents an example indicating possible economic interpretations of smoothness. ${ }^{19}$

EXAMPLE 4.5: We present here an example of a smooth type space that indicates that the result of Theorem A.1, and indeed the concept of smoothness itself, have economic interpretations.

Robert is in the United States. William is in the United Kingdom. Mario in Italy, a common friend, wishes to transfer to either Robert or William his subscription to Econometrica, for which he paid $€ C$. Assume $€ 1=\$ e^{\alpha}=£ e^{\beta}$. Mario converts $€ C$ into the other currencies and sends these figures to Robert and William. However, Mario tends to be somewhat scattered, and with probability $\varepsilon$ may have confused the numbers, that is, with $1-\varepsilon$ probability Robert receives the figure $\$ C \cdot e^{\alpha}$ and William receives the figure $£ C \cdot e^{\beta}$, which is correct, but with $\varepsilon$ probability Robert receives the figure $\$ C e^{\beta}$ and William receives the figure $£ C e^{\alpha}$. Both Robert and William are aware of this possibility of confusion on the part of Mario.

We may model the knowledge space as $\Omega^{\prime}=\mathbb{R}_{++} \times \mathbb{R}_{++} \times \mathbb{R}_{++}$, specifying Mario's price, and the figures received by Robert and William, respectively. However, note that if the figures Robert $\omega_{1}$ and William $\omega_{2}$ are revealed, one could deduce whether Mario mixed up or not, simply by checking whether $\frac{\omega_{1}}{\omega_{2}}=e^{\alpha-\beta}$ or $=e^{\beta-\alpha}$, hence deducing Mario's price. We will therefore model the knowledge space using $\Omega=\mathbb{R}_{++} \times \mathbb{R}_{++}$for specifying Robert's and William's figures.

The classes of $\mathcal{E}^{R}$, representing Robert's knowledge, are $\left(\left\{\left(r, e^{\alpha-\beta} \cdot r\right),\left(r, e^{\beta-\alpha} \cdot r\right)\right\}\right)_{r \in \mathbb{R}_{++}}$, and the classes of $\mathcal{E}^{W}$, representing William's knowledge, are $\left(\left\{\left(e^{\alpha-\beta} \cdot r, r\right),\left(e^{\beta-\alpha}\right.\right.\right.$. $r, r)\})_{r \in \mathbb{R}_{++}}$. The beliefs are given by

$$
t_{\left(r, e^{\alpha-\beta, r)}\right.}^{R}[\omega]=t_{\left(r, e^{\beta-\alpha, r)}\right.}^{R}[\omega]= \begin{cases}1-\varepsilon & \text { if } \omega=\left(r, r \cdot e^{\beta-\alpha}\right), \\ \varepsilon & \text { if } \omega=\left(r, r \cdot e^{\alpha-\beta}\right), \\ 0, & \text { otherwise, }\end{cases}
$$

and similarly for $t^{W}$.

One then easily sees that an equivalence class $C$ of the relation $\mathcal{E}$ induced by $\mathcal{E}^{W}, \mathcal{E}^{R}$ is of the form

$$
C=\left\{r \cdot e^{2 n \gamma} \cdot\left(1, e^{\gamma}\right) \mid n \in \mathbb{Z}\right\} \cup\left\{r \cdot e^{2 n \gamma} \cdot\left(1, e^{-\gamma}\right) \mid n \in \mathbb{Z}\right\}
$$

for some $r \in \mathbb{R}_{++}$, where $\gamma=\alpha-\beta$. This relation is seen to be smooth, by applying Theorem A.1: each of the two sets of which $C$ is expressed as the union clearly consists only of points isolated within itself.

\subsection{Bayesian Games and Bayesian Equilibria}

A Bayesian game $\Gamma=(\Omega, I, t, A, r)$ consists of the following components:

- $(\Omega, I, t)$ forms a type space (with knowledge relations $\mathcal{E}^{i}$ understood implicitly as generated by the types $t$ ).

- $A=\left(A^{i}\right)_{i \in I}$ is a tuple consisting of a finite action set for each player $i \in I$.

\footnotetext{
${ }^{19}$ Examples of non-smooth type spaces can be found in Hellman (2014b), Simon (2003), and Lehrer and Samet (2011); we do not present such examples here, as our focus is on smooth type spaces.
} 
- $r: \Omega \times \prod_{i \in I} A^{i} \rightarrow \mathbb{R}^{I}$ is a bounded measurable payoff function, with $r^{i}$ then being the resulting payoff to player $i$. The payoff function $r$ extends multi-linearly to mixed actions in the usual manner.

DEFINITION 4.6: A Bayesian game is purely atomic if the underlying type space is purely atomic. A Bayesian game is smooth if the underlying type space is smooth.

A strategy of a player $i \in I$ is a mapping $s^{i}: \Omega \rightarrow \Delta\left(A^{i}\right)$ which is constant on each player's knowledge component. In other words, if $\omega, \omega^{\prime} \in \Omega$ are in the same partition element of $\mathcal{E}^{i}$, then $s^{i}(\omega)=s^{i}\left(\omega^{\prime}\right)$.

A Bayesian equilibrium is a profile of strategies $s=\left(s^{i}\right)_{i \in I}$ such that for each $i \in I$, every $\omega \in \Omega$, and each alternative strategy $x \in \Delta\left(A^{i}\right)$ of player $i$,

$$
\sum_{\left\{\omega^{\prime} \mid\left(\omega^{\prime}, \omega\right) \in \mathcal{E} \mathcal{E}^{i}\right\}} r^{i}\left(\omega^{\prime}, s\left(\omega^{\prime}\right)\right) \pi_{\omega}^{i}\left[\omega^{\prime}\right] \geq \sum_{\left\{\omega^{\prime} \mid\left(\omega^{\prime}, \omega\right) \in \mathcal{E}^{i}\right\}} r^{i}\left(\omega^{\prime}, x, s^{-i}\left(\omega^{\prime}\right)\right) \pi_{\omega}^{i}\left[\omega^{\prime}\right] .
$$

When a Bayesian equilibrium $s$ satisfies the condition that each $s^{i}$ is Borel-measurable, $s$ is said to be a measurable ${ }^{20}$ Bayesian equilibrium. (Hence, $s^{i}$ is $\sigma\left(\mathcal{E}^{i}\right)$-measurable.)

\subsection{Existence of Measurable Bayesian Equilibrium}

We show how the following, appearing in ${ }^{21}$ Hellman and Levy (2017), follows from the above selection theorem, Theorem 3.1.

THEOREM 4.7: If the common knowledge equivalence relation $\mathcal{E}$ of the type space underlying a purely atomic Bayesian game is smooth, then the game admits a measurable Bayesian equilibrium.

Proof: Fix a Bayesian game $\left(\Omega,\left(t^{i}\right)_{i \in I},\left(r^{i}\right)_{i \in I}\right)$. Fix a finite or countable set $S$, such that all common knowledge equivalence relations are of cardinality $S$; the case in which not all classes are of the same cardinality follows by Proposition 3.4. We use the language $\mathcal{L}=(+, \cdot,>,=, 0,1)$, where,$+ \cdot$ are 2 -place function symbols, $>,=$ are 2 -place relation symbols, and 0,1 are constants. The first (and main) step is to define, in the language $\mathcal{L}_{\omega_{1}, \omega}$, a (quantifier-free) formula whose free variables are the beliefs, payoffs, and strategies of Bayesian games with state space $S$, which expresses the fact that the given strategies are an equilibrium of the game with given payoffs and beliefs. To simplify notation, it will be understood that $s, t, u, v$ are indices in $S$ (to be thought of as the cardinality of common knowledge equivalence classes), while $i, j$ represent players in $I, a^{i}$ is the action of a player $i$ in $A^{i}$, and $a=\left(a_{1}, \ldots, a_{n}\right)$ is a profile of actions in $\prod_{i} A^{i}$.

- For $j \in I$ and $u, v \in S$, the variable $\tau_{j, u, v}$ represents player $j$ 's belief about state $v$ in state $u$.

- For $j \in I, t \in S$, and $a \in \prod_{i} A^{i}$, the variable $g_{j, t, a}$ represents player $j$ 's payoff at state $t$ given the action profile.

- For $j \in I, t \in S$, and $a_{j} \in A^{j}$, the variable $x_{j, t, a^{j}}$ represents the weight player $j$ puts on his action $a^{j}$ in state $t$.

\footnotetext{
${ }^{20}$ It is possible for a game to have Bayesian $\varepsilon$-equilibria that are not measurable as in, for example, Simon (2003) or Hellman (2014b).

${ }^{21}$ That paper assumed positivity of the type space, although as remarked there it is not a crucial assumption. Here this assumption is not used at all for this theorem.
} 
Formally, in the framework and notation of Section 3.1, we would have the various dimensions of the input data $\bar{F}_{D}=\left(N_{1}, \ldots, N_{K}\right)=(2, \ldots, 2,1, \ldots, 1)$, where $N_{j}=2$ for $j \leq|I|, N_{j}=1$ for $|I|<j \leq K:=|I|+|I| \times_{i}\left|A^{i}\right|$, as there are $2 \times S \times S$ variables to specify the beliefs of each of the $I$ players, and there are $S$ variables to specify the payoffs for each player for each action profile. $F_{U}=L=\sum_{i}\left|A^{i}\right|$ and $\bar{F}=(1, \ldots, 1) \in \mathbb{R}^{L}$, as there are $S$ variables to specify over all states the weight to give a specific action for a specific player. We now construct the formula $\alpha$ :

- $\sum\left(x_{s}\right) \geq \sum\left(y_{s}\right)$ (which we write instead of the more notationally cumbersome $\left.R\left(\left(x_{s}\right),\left(y_{s}\right)\right)\right)$, which holds in $\mathbb{R}$ if and only if $\left(x_{s}\right),\left(y_{s}\right)$ are summable and $\sum_{s} x_{s} \geq \sum_{s} y_{s}$; such a formula can easily be written in a quantifier-free formulation in the spirit of Example B.3.

- For each $i=1, \ldots, I$, define $\beta^{i}\left(\left(x_{a^{i}}\right)\right)$, which holds if and only if $\left(x_{a^{i}}\right) \in \Delta\left(A^{i}\right)$, that is, $\left(x_{a^{i}}\right)$ represents a mixed action of player $i$ :

$$
\beta^{i}\left(\left(x_{a^{i}}\right)\right)=\left(\sum_{a^{i} \in A^{i}} x_{a^{i}}=1\right) \bigwedge_{a^{i} \in A^{i}}\left(x_{a^{i}} \geq 0\right) .
$$

- For each $j=1, \ldots, I$ and $s, t \in S$, define

$$
\eta_{j, s, t}\left(\left(\tau_{j, u, v}\right)_{u, v}\right)=\bigwedge_{u}\left(\tau_{j, s, u}=\tau_{j, t, u}\right),
$$

which is understood as holding if player $j$ has the same beliefs at state $s$ as in state $t$.

$$
\begin{aligned}
\beta\left(\left(x_{i, t, a^{i}}\right),\left(\tau_{i, u, v}\right)\right)= & {\left[\bigwedge_{i} \bigwedge_{t} \beta^{i}\left(\left(x_{t, i, a^{i}}\right)\right)\right] } \\
& \wedge\left[\bigwedge_{i} \bigwedge_{s, t}\left(\eta_{i, s, t}\left(\tau_{j, u, v}\right)_{u, v}\right) \rightarrow \bigwedge_{a^{i}}\left(x_{i, s, a^{i}}=x_{i, t, a^{i}}\right)\right],
\end{aligned}
$$

which holds if and only if mixed actions are played at each state (which is what the $\bigwedge_{i} \bigwedge_{t} \beta^{i}\left(\left(x_{t, i, a^{i}}\right)\right)$ term just after the equality sign means), and strategies are measurable with respect to player $i$ 's knowledge (which is what the term at the bottom line means).

- For each $j \in I$, let

$$
\begin{aligned}
& \gamma_{j, t}\left(\left(g_{j, t, a}\right),\left(x_{t, i, a^{i}}\right),\left(\tau_{i, t, u}\right)\right) \\
& =\bigwedge_{b \in A^{j}}\left(\sum_{u} \sum_{a} \tau_{t} \cdot g_{j, t, a} \prod_{i} x_{t, i, a^{i}} \geq \sum_{u} \sum_{a} \tau_{t} \cdot g_{j, t, b, a_{-i}} \prod_{i \neq j} x_{t, i, a^{i}}\right),
\end{aligned}
$$

which is understood as it not being profitable for player $j$ in state $t$ to switch to any action $b$ when beliefs are $\left(\tau_{j, t, u}\right)$, his payoff at state $t$ under action profile $a$ in $g_{j, t, a}$, and at state $t$ player $i$ places weight $x_{t, i, a^{i}}$ on action $a^{i}$ in state $t$.

- Finally, let

$$
\begin{aligned}
\alpha & \left(\left(g_{j, t, a}\right),\left(x_{t, i, a^{i}}\right),\left(\tau_{j, t, u}\right)\right) \\
& =\bigwedge_{j} \bigwedge_{t} \gamma_{j, t}\left(\left(g_{j, t, a}\right),\left(x_{t, i, a^{i}}\right),\left(\tau_{j, t, u}\right)\right) \wedge \beta\left(\left(x_{i, t, a^{i}}\right),\left(\tau_{i, t, u}\right)\right),
\end{aligned}
$$


which is understood as $\gamma_{j, t}$ holding when the payoffs are given by $j$ 's payoffs and for $j$ 's belief at state $t$, for each $j \in I$ and $t \in S$, as well as the strategies being legitimate and functions of only each player's knowledge.

The determined variables here are

$$
F_{D}=\left\{\left(\tau_{j, t, u}\right)_{t, u \in S, j \in I},\left(g_{j, t, a}\right)_{t \in S, j \in I, a \in \prod_{i} A^{i}}\right\},
$$

that is, the beliefs of the players and the payoffs, which are regarded as the 'givens' of a Bayesian game, while the undetermined variables are the strategies that the players may choose,

$$
F_{U}=\left(x_{t, i, a^{i}}\right)_{t \in S, i \in I, a^{i} \in A^{i}} .
$$

Continuing along the lines of the general description of Section $3, \zeta_{j, a}(\omega)$ here is the payoff at state $\omega$ of player $j$ when action profile $a$ is played, for each $j$ and profile $a$, and $\zeta_{k}(\omega, \theta)$ is the belief of player $k$ at state $\omega$ about the probability of state $\theta$, for each $k$. Then, by Equation (3.1), the mapping $\zeta$ determines these variables of the model, given by the components $\left(r^{j}(\cdot, a)\right)$ and $\left(\pi^{j}(\cdot)[\cdot]\right)$ :

$$
\zeta_{k}(\omega, \theta)=\pi_{\omega}^{k}(\theta), \quad \zeta_{j, a}(\omega)=r^{j}(\omega, a) .
$$

Recalling Equation (3.2), namely $Z=\left\{x \in \Phi^{F_{D}} \times \Phi^{F_{U}} \mid \alpha(x)\right.$ holds $\}$, where in this case $\alpha$ is the statement that it is not profitable for any player to switch to any other action at any state, we have that $Z$ is Borel by Proposition 3.6.

Furthermore, following the definition in Equation (3.3), for every equivalence class $C$ of $\mathcal{E}$ and any bijection $\phi$ of $C$ with $S$, the set $Z[\zeta, \phi]$ is precisely all the Bayesian equilibria of the Bayesian game over the image of the state space $C$ under $\phi$, with payoffs and beliefs induced by $\phi: C \rightarrow S$ and $\zeta$. Every Bayesian game with a countable state space possesses equilibria (see, e.g., Simon (2003, Proposition 1)), and furthermore, the set of equilibria is compact in the Tychonoff topology. Since $S$ is at most countable, it follows that $Z[\zeta, \phi] \neq \emptyset$ and is compact.

We now have all the pieces for applying Theorem 3.1(b). That is, for a Bayesian game over a smooth type space, there exists a Borel-measurable mapping $\Phi: \Omega \rightarrow S$ s.t. for each class $C,\left.\Phi\right|_{C}$ is a bijection between $C$ and $S$, and a Borel-measurable $\Psi$, whose domain is the collection of equivalence classes of $\mathcal{E}$ (i.e., the common knowledge components of the game), such that the mapping $\Psi$ selects, for each common knowledge component $C$, one Bayesian equilibrium over $S$, with the payoffs and beliefs induced by the payoffs and beliefs on $C$. Formally, for each equivalence class $C$, it holds that in the game on state space $S$, where, for player $i$, payoffs in state $s$ under action profile $a$ are $r^{i}\left(\left.\Phi\right|_{C} ^{-1}(s), a\right)$ and belief in state $s$ about state $t$ is $\pi_{\left.\Phi\right|_{C} ^{-1}(s)}^{i}\left(\left.\Phi\right|_{C} ^{-1}(t)\right)$, the profile in which, in state $s$, player $i$ places on action $a^{i}$ weight $\Psi_{i, s, a^{i}}(C)$ is an equilibrium.

The selection of an equilibrium under $\Psi$ for each component is the crucial step towards the 'gluing' of these equilibria into one global, measurable equilibrium. Fomally, define for each player $j \in I$ and each action $a^{j} \in A^{j}$,

$$
\sigma^{j}(\omega)\left[a^{j}\right]=\Psi_{j, \Phi(\omega), a^{i}}(\iota(\omega)),
$$

where $\iota: \Omega \rightarrow \Omega / \mathcal{E}$ is the quotient map. This is the measurable Bayesian equilibrium we seek, relating the probability of player $j$ choosing action $a^{j}$ at state $\omega \in \Omega$ to the corresponding value in the equilibrium selected by $\Psi$ in the common knowledge component containing $\omega$.

Q.E.D. 
REMARK 4.8: The proofs of several theorems in this paper will follow the general lines of the proof of Theorem 4.7. As mentioned above, the key in many of the theorems here, relating to some game over an uncountable state space, involves showing that this state space can be considered to be composed of disjoint countable sets, each of which is 'naturally' identified as the equivalence class of a Borel equivalence relation, and furthermore, each such countable Borel equivalence class can be identified with at least one measurable equilibrium.

Then if the defining countable Borel equivalence relation is smooth, all we will need to do is to construct a formula $\alpha$ in an $\mathcal{L}_{\omega_{1}, \omega}$ language that 'witnesses' the existence of an equilibrium over the countable Borel equivalence classes and follow the steps of the proof of Theorem 4.7, mutatis mutandis. In several subsequent proofs, we will therefore be satisfied with the task of constructing such a formula and rely on the rest of the proof being obvious from that point onwards, pointing to the proof of Theorem 4.7 as showing the way to the completion of the proof.

\section{STOCHASTIC GAMES WITH COUNTABLE ORBITS}

\subsection{The Stochastic Games Model}

The components of a discounted stochastic game with a continuum of states and finitely many actions are the following:

- A Polish space $\Omega$ of states.

- A finite set $I$ of players.

- A finite set of actions $A^{i}$ for each $i \in I$.

- A discount rate $\lambda \in(0,1)$.

- A bounded payoff function $r: \Omega \times \prod_{i} A^{i} \rightarrow \mathbb{R}^{I}$, which is Borel-measurable.

- A transition function $q: \Omega \times \prod_{i} A^{i} \rightarrow \Delta(\Omega)$, which is Borel-measurable. ${ }^{22}$

The game is played in discrete time. If $z \in \Omega$ is a state at some stage of the game and the players select an action profile $a \in \prod_{i} A^{i}$, then $q(z, a)$ is the conditional (given the past) probability distribution of the next state of the game. A stationary strategy for player $i$ is a behavioral strategy that depends only on the current state; equivalently, it is a Borelmeasurable mapping that associates with each state $z \in \Omega$ a probability distribution on the set $A^{i}$.

For any profile of behavioral strategies $\sigma=\left(\sigma^{i}\right)_{i \in I}$ of the players and every initial state $z_{1}=z \in \Omega$, a probability measure $P_{z}^{\sigma}$ and a stochastic process $\left(z_{n}, a_{n}\right)_{n \in \mathbb{N}}$ are defined on $H^{\infty}:=\left(\Omega \times \prod_{i \in I} A^{i}\right)^{\mathbb{N}}$ in a canonical way, where the random variables $z_{n}, a_{n}$ describe the state and the action profile chosen by the players, respectively, in the $n$th stage of the game (see, e.g., Bertsekas and Shreve (1996)). The (unnormalized) $\lambda$-discounted expected payoff vector under $\sigma$, in the game starting from state $z$, is

$$
\gamma_{\sigma}^{\lambda}(z)=E_{z}^{\sigma}\left(\sum_{n=1}^{\infty}(1-\lambda)^{n-1} r\left(z_{n}, a_{n}\right)\right) .
$$

Let $\Sigma^{i}$ denote the set of behavioral strategies for player $i \in I$. A profile $\sigma \in \prod_{i \in I} \Sigma^{i}$ will be called a Nash equilibrium of the ( $\lambda$-discounted) stochastic game if

$$
\gamma_{\sigma}^{i}(z) \geq \gamma_{\left(\tau, \sigma^{-i}\right)}^{i}(z), \quad \forall i \in I, \forall z \in \Omega, \forall \tau \in \Sigma^{i} .
$$

\footnotetext{
${ }^{22}$ Where $\Delta(\Omega)$, the space of regular Borel probability measures on $\Omega$, possesses the Borel structure induced from the topology of narrow convergence.
} 
It is well known that a stationary profile $\sigma$ is an equilibrium iff for all $z \in \Omega, \sigma(z)$ is an equilibrium in the game with payoffs:

$$
G_{\sigma}(z, a):=r(z, a)+(1-\lambda) \int_{\Omega} \gamma_{\sigma}\left(z^{\prime}\right) q\left(d z^{\prime} \mid z, a\right) .
$$

\subsection{Countable Atomic Orbits}

DEFINITION 5.1: A stochastic game is purely atomic if it has countable orbits, meaning that:

- For each state $z \in \Omega$ and every action profile $a \in A$, the transition measure $q(z, a)$ is a purely atomic measure. Denote

$$
Q(z):=\left\{z^{\prime} \in \Omega \mid \exists a \in A, q\left(z^{\prime} \mid z, a\right)>0\right\} .
$$

- For each $z \in \Omega$, the set

$$
Q^{-1}(z)=\left\{z^{\prime} \in \Omega \mid \exists a \in A, q\left(z \mid z^{\prime}, a\right)>0\right\}
$$

is countable.

That is, from each state, there are at most countably many states it can go on to, and at most countably many states from which it could have arrived. We use this to define an equivalence relation whose equivalence classes are the orbits of $q$, namely, the transitive, reflexive, and symmetric closure of $Q$. Under the above assumptions, each orbit is countable.

When $\Omega$ is itself countable, the game clearly has countable orbits. When $\Omega$ is countable, it is well known that the game has stationary equilibria (see Parthasarathy (1973)). Levy (2013b) showed, however, that when the state space of a stochastic game is uncountable, even if the game has countable orbits, ${ }^{23}$ it may not possess (measurable) stationary equilibria. $^{24}$

A purely atomic stochastic game with countable orbits in which the orbit equivalence relation, as defined in Definition 5.1, is smooth may be termed a smooth stochastic game. Smooth stochastic games can arise naturally in economic models, as the next example shows.

In contrast to the negative result of the counter-example in Levy (2013b), the following theorem provides a sufficient condition for the existence of measurable stationary equilibria in a stochastic game with an uncountable state space.

THEOREM 5.2: A purely atomic stochastic game in which the orbit equivalence relation is smooth admits a measurable stationary equilibrium.

PROOF: The proof is similar to that of Theorem 4.7, as per Remark 4.8. Again, by Proposition 3.4, it suffices to consider the case when all orbits are of the same cardinality

\footnotetext{
${ }^{23}$ The game in Levy (2013b) technically has an absorbing state with payoff 0 that can be reached from any state, making the orbits not strictly countable, but the single absorbing state can be replaced with a set of absorbing states; alternatively, see the related notion of $\mathcal{S}$-countable orbits below.

${ }^{24} \mathrm{~A}$ different example of a stochastic game lacking equilibria appears in Levy and McLennan (2015), but in that case all transitions are absolutely continuous with respect to a fixed measure; in particular, the transitions there are not purely atomic.
} 
$S$. We use the language $\mathcal{L}=(+, \cdot,>,=, 0,1, \lambda)$, much like in the earlier, but now add the discount factor to the language, which has the assignment in $(0,1)$ of the game's discount factor; over the parameter space $X=\mathbb{R}$, these functions and relations have the usual meanings. We again define a quantifier-free formula in the language $\mathcal{L}_{\omega_{1}, \omega}$, where again the indices $s, t$ are understood to be in $S ; i, j$ are indices of players in $I ; a^{i}$ is an action in $A^{i}$; and $a$ is a profile of actions in $\prod_{i} A^{i}$.

- The relations $\sum(\cdot) \geq \sum(\cdot), \beta^{1}, \ldots, \beta^{I}$ are as defined in the proof of Theorem 4.7, where $\sum_{s} x_{s} \geq \sum_{s} y_{s}$ denotes (instead of a more cumbersome $\left.R\left(\left(x_{s}\right)_{s},\left(y_{s}\right)_{s}\right)\right)$ the summability of both series and the inequality of the sums, and $\beta^{i}\left(\left(x_{a_{i}}\right)_{a_{i} \in A^{i}}\right)$ defined in (4.2) denotes that $\left(x_{a_{i}}\right)_{a_{i} \in A^{i}}$ is a mixed action of player $i$; also define similarly $\sum(\cdot)=\sum(\cdot)$. We similarly write $v=\sum_{t} x_{t}$ to express that fact.

- Let $g_{s, i, a}$ be interpreted as the payoff to player $i$ at state $s$ when $a$ is the action profile, $q_{s, t, a}$ be interpreted as the probability of transition to state $t$ when action profile $a$ is selected in state $s, x_{t, i, a^{i}}$ be the wait player $i \in I$ places on action $a^{i} \in A^{i}$ in state $t \in S$, and $v_{i, t}$ denote the payoff for player $i$ in the game beginning in state $t$.

- Let

$$
\begin{aligned}
& \rho\left(\left(v_{i, s}\right),\left(g_{s, i, a}\right),\left(x_{s, i, a^{i}}\right),\left(q_{s, t, a}\right)\right) \\
& =\bigwedge_{i} \bigwedge_{s}\left(v_{i, s}=\sum_{a}\left(\prod_{i \in I} x_{s, i, a^{i}}\right)\left(g_{s, i, a}+(1-\lambda) q_{s, t, a} v_{i, t}\right)\right)
\end{aligned}
$$

state that at each state $s \in S$, the payoffs under the mixed action profile $\left(x_{s, i, a^{i}}\right)$ of the auxiliary game induced by the dynamic program is $v_{i, s}$ for player $i$.

- Let

$$
\begin{aligned}
\eta & \left(\left(v_{i, s}\right),\left(g_{s, i, a}\right),\left(x_{s, i, a^{i}}\right),\left(q_{s, t, a}\right)\right) \\
& =\bigwedge_{i} \bigwedge_{s} \bigwedge_{b \in A^{i}}\left[v_{i, s} \geq \sum_{t} \sum_{a}\left(\prod_{j \neq i} x_{s, j, a^{j}}\right)\left(g_{s, j, b, a_{-i}}+(1-\lambda) q_{s, t, b, a_{-i}} v_{j, t}\right)\right]
\end{aligned}
$$

state that at each state $s \in S$, no deviation in the auxiliary game induced by the dynamic program with continuation payoff $\left(v_{i, t}\right)$ yields payoff no more than $v_{i, s}$.

- Finally, let

$$
\begin{aligned}
\alpha\left(\left(v_{i, s}\right),\left(g_{s, i, a}\right),\left(x_{s, i, a^{i}}\right),\left(q_{s, t, a}\right)\right) & \\
= & \bigwedge_{j \in I} \bigwedge_{s \in S} \beta^{j}\left(\left(x_{s, j, a^{j}}\right)\right) \\
& \wedge \rho\left(\left(v_{i, s}\right),\left(g_{s, i, a}\right),\left(x_{s, i, a^{i}}\right),\left(q_{s, t, a}\right)\right) \\
& \wedge \eta\left(\left(v_{i, s}\right),\left(g_{s, i, a}\right),\left(x_{s, i, a^{i}}\right),\left(q_{s, t, a}\right)\right),
\end{aligned}
$$

that is, each player is playing a probability distribution in each state, and the profiles in state $s$ give the right payoffs using equilibrium strategies.

The rest of the proof follows the same lines (see Remark 4.8) as the proof of Theorem 4.7, relying on the fact that, as already mentioned, stochastic games with countable state spaces possess stationary equilibria (Parthasarathy (1973)). 


\subsection{Countable Orbits With Respect to Finite $\pi$-Systems}

Say that a subset $S \subseteq \Omega$ is $Q$-invariant if $Q(z) \subseteq S$ for all $z \in S$. Recall that a $\pi$-system $\mathcal{S}$ is a non-empty collection of subsets closed under finite intersection. Given a finite $\pi$ system $\mathcal{S}$ on $\Omega$ of $Q$-invariant sets, for each $S \in \mathcal{S} \cup\{\Omega\}$, denote

$$
\hat{S}=S \backslash S_{0}, \quad \text { where } S_{0}=\bigcup\left\{S^{\prime} \mid S^{\prime} \in \mathcal{S}, S^{\prime} \subseteq S, S^{\prime} \neq S\right\} .
$$

Define the $S$-orbit equivalence relation $\mathcal{O}_{S}$ on $S$ (actually, on $\hat{S}$ ) to be the reflexive, symmetric, and transitive closure of the relation $Q \cap(\hat{S} \times \hat{S})$. From this, define the $\mathcal{S}$-orbit equivalence relation $\mathcal{O}_{\mathcal{S}}=\bigcup_{S \in \mathcal{S} \cup\{\Omega\}} \mathcal{O}_{S}$.

Intuitively, for each element $S$ of a $\pi$-system $\mathcal{S}$ ( or $S=\Omega$ ), let $S_{0}$ denote the union of all $\mathcal{S}$ elements strictly contained in $S$. (Note that $S_{0}$ is also $Q$-invariant.) Then two elements $\omega_{1}, \omega_{2}$ which are in $\hat{S}:=S \backslash S_{0}$-that is, in $S$ but not in $S_{0}$-are in the same $S$-orbit if and only if there is positive probability of eventually reaching some state $\omega_{3}$ also in $\hat{S}$ from either $\omega_{1}$ or $\omega_{2}$, by some selections of actions. In other words, $S$-orbit equivalence is like regular orbit equivalence, but it takes into account only what happens in $S$ without entering $S_{0}$.

We will say that a game has $\mathcal{S}$-countable orbits if all the $\mathcal{S}$-orbits are countable.

We will next show that if the game has $\mathcal{S}$-countable orbits and the $\mathcal{S}$-orbit relation is smooth, then there is always a measurable stationary equilibrium over the entire state space. The idea is that it suffices first to find equilibria in the game restricted to each $\subseteq$ minimal element of such a collection $\mathcal{S}$, and then to do so inductively for larger elements of $\mathcal{S}$ until finally one attains a measurable equilibrium for all $\Omega$. The proof is given in the Appendix.

THEOREM 5.3: If there is a finite $\pi$-system $\mathcal{S}$ of $Q$-invariant sets such that the game has $\mathcal{S}$-countable orbits, and the $\mathcal{S}$-orbit relation is smooth, then it admits a measurable stationary equilibrium.

EXAMPLE 5.4: $N$ players are gambling. At each stage, a player who chooses to enter must bet his entire wealth, and payoffs from each bet are always double-or-nothing. The actions of the agents (as well as perhaps the wealth held by the agents at that round) affect the odds of each player doubling his money or losing everything.

The state space is taken to be $\Omega=\mathbb{R}_{+}^{N}$, the nonnegative $N$-tuples of real number. The transitions are such that

$$
q\left(\underset{i=1}{X}\left\{0, x^{i}, 2 x^{i}\right\} \mid\left(x^{1}, \ldots, x^{N}\right),\left(a^{1}, \ldots, a^{N}\right)\right)=1
$$

for all wealth levels $x^{1}, \ldots, x^{N}$, and all actions $a^{1}, \ldots, a^{N}$. Now, for each $K \subseteq N$, define

$$
\Omega_{K}=\left\{\left(x^{1}, \ldots, x^{N}\right) \mid \forall j \in K, x^{j}=0\right\},
$$

which are easily seen to be $Q$-invariant (in particular, $\Omega_{\emptyset}=\Omega, \Omega_{N}=\{(0, \ldots, 0)\}$ ). Let $\mathcal{S}$ be the $\pi$-system $\left\{\Omega_{K} \mid K \subseteq N\right\}$; indeed, $\Omega_{K} \cap \Omega_{K^{\prime}}=\Omega_{K \cup K^{\prime}}$. We see then that every $\mathcal{S}$ orbit is of the form

$$
\left\{\left(x^{1}, \ldots, x^{N}\right) \mid \forall j \in K, x^{j}=0 \text { and } \forall j \notin K, \exists n^{j} \in \mathbb{Z}, \text { such that } x^{j}=a^{j} \cdot 2^{n^{j}}\right\}
$$


for some $K \in N$ and some $\left(a^{j}\right)_{j \notin K} \in \mathbb{R}^{N-K}$; this orbit is contained in $\Omega_{K}$. The smoothness of the relation induced by these orbits follows from Theorem A.1 of the Appendix (indeed, a limit point of an orbit in $\Omega_{K}$ would be a point in $\Omega_{K^{\prime}}$ for some $K^{\prime}$ strictly larger than $K$ ) and hence the game possesses a smooth $\mathcal{S}$-orbit relation. As a result, Theorem 5.3 shows that if the transitions of a stochastic game satisfy (5.3), then the game possesses a stationary equilibrium, without the need to impose further assumptions on transitions or payoffs.

A further application of Theorem 5.3 is given in Section 6 .

\section{REPEATED GAMES WITH SYMMETRIC INCOMPLETE INFORMATION}

The following games were introduced in Kohlberg and Zamir (1974) in the case of deterministic signaling in zero-sum games, and generalized to random signaling in zero-sum games by Forges (1982). For the extension to the non zero-sum case, which is relevant to the presentation here and other developments, see Neyman and Sorin (2003, Chapter 21) and the references therein. For a general model of repeated games, see Mertens, Sorin, and Zamir (2015, Chapter IV).

We first present an economic example of a repeated game with symmetric incomplete information.

In the model here, there are a finite $K$ many $G^{1}, \ldots, G^{K} I$-player strategic form games, with finite action space $A^{i}$ for each player $i \in I$. A game is chosen, once and for all, at the start of play according to a prior $p \in \Delta(K)$ that is known to the players, but the game is not revealed to the players. The game is played repeatedly, with each player observing the actions of the other players. In addition, following a stage at which a profile $a \in \prod_{i} A^{i}$ was played, a signal from a finite set $M$ is received according to a distribution $q_{k}(a)$ on $M$ when the $k$ th game was chosen.

This can be modeled naturally as a stochastic game with a continuum of states, where the state space is $\Omega=\Delta(K)$, the simplex of possible probability distributions over the $K$ games. If at any point in time the beliefs of the players about the true game are given by $p \in \Delta(K)$ and a profile of actions $a$ is played leading to a signal $m$, the new belief $p^{\prime}$ of the players is obtained via Bayesian updating as

$$
p_{k}^{\prime}=\frac{p_{k} \cdot q_{k}(m \mid a)}{\sum_{l=1}^{K} p_{l} \cdot q_{l}(m \mid a)}, \quad k=1, \ldots, K .
$$

Hence, the transitions in the auxiliary game, which by mildly abusive notation we will also denote $q$, can be written (by $\sum E$ we mean $\sum_{e \in E} e$ )

$$
\begin{array}{r}
q\left(p^{\prime} \mid p, a\right)=\sum\left\{\sum_{k \in K} q_{k}(m \mid a) \cdot p_{k} \mid m \in M\right. \\
\text { s.t. } \left.p^{\prime}=\left(\frac{p_{k} \cdot q_{k}(m \mid a)}{\sum_{l=1}^{K} p_{l} \cdot q_{l}(m \mid a)}\right)_{k \in K}\right\} .
\end{array}
$$


Note that this probability is 0 (as the sum is empty) except for finitely many (in fact, at most $|M|$ many) values of $p^{\prime}$. Hence, the auxiliary state change follows a stochastic games dynamic. The payoffs, as a function of the new state in the game, are now given by

$$
r(p, a)=\sum_{k=1}^{K} p_{k} G^{k}(a) .
$$

Given a discount rate $\lambda$, it can be shown (see references above) that a measurable stationary equilibrium $\sigma$ of the $\lambda$-discounted auxiliary stochastic game induces an equilibrium in the repeated game which depends only on the current common state belief in the obvious way. Furthermore, if we let $\mathcal{S}=\{\Delta(L) \mid \emptyset \neq L \subseteq K\}$, then it is easy to see that the game has $\mathcal{S}$-countable orbits.

If the game is a two player zero-sum game, then it is known that a measurable equilibrium exists, as discounted zero-sum games with general state spaces possess measurable value and optimal strategies; see, for example, Parthasarathy (1972, Chapter 4). For general-sum games, the matter is open, since, as remarked earlier, discounted generalsum stochastic games need not in general possess measurable equilibria. Furthermore, the auxiliary game on the simplex in general, and in fact generically, ${ }^{25}$ does not possess smooth $\mathcal{S}$-orbits (when $\mathcal{S}$ is the set of faces of the simplex); this is shown in Hellman and Levy (2019).

\subsection{Analysis of an Example}

Suppose the signal space is $M=K \cup\{\emptyset\}$ and that there are mappings $\mu_{1}, \ldots, \mu_{k}$ : $\prod_{i} A^{i} \rightarrow(0,1)$ such that for all action profiles $a \in \prod_{i} A^{i}$,

$$
q_{k}(k \mid a)=1-\mu_{k}(a), \quad q_{k}(\emptyset \mid a)=\mu_{k}(a) .
$$

That is, with probability $1-\mu_{k}$, it is announced that the true state is some state $k$, and with probability $\mu_{k}$, the signal is $\emptyset$. We will make the strong assumption that $1>\mu_{K}>\cdots>$ $\mu_{1}>0$ for all action profiles. If $\mathcal{S}$ denotes the vertices of $\Delta(K)$, then by Equation (6.1) the game has $\mathcal{S}$-countable orbits. We contend that under these assumptions, the $\mathcal{S}$-orbit relationship is smooth. Denote

$$
\delta=\min \left\{\mu_{j+1}(a)-\mu_{j}(a) \mid a \in \prod_{i} A^{i}, j=1, \ldots, K-1\right\}>0 .
$$

Define $f: \Delta(K) \times \prod_{i} A^{i} \rightarrow \Delta(K)$ by

$$
f(p, a)=\left(\frac{p_{k} \mu_{k}(a)}{\sum_{l} p_{l} \mu_{l}(a)}\right)_{k \in K} .
$$

In this case, transition in the auxiliary game from state $p$ under action profile $a$ is to $f(p, a)$ or to one of the pure states, with probabilities given by

$$
q(f(p, a) \mid p, a)=\sum_{\ell} p_{\ell} \mu_{\ell}(a), \quad q\left(\delta_{l} \mid p, a\right)=\left(1-\mu_{l}(a)\right) p_{l} .
$$

\footnotetext{
${ }^{25}$ That is, for a collection of $\left(q_{k}(a)\right)_{k, a, m}$ of Lebesgue-null and meager complement in $(\Delta(M))^{K \times A}$.
} 
Denote $F(p):=\left\{f(p, a) \mid a \in \prod_{i} A^{i}\right\}$. Fixing some $p_{0} \in \Delta(K)$, which is not a pure state, denote by $L$ the support of $p_{0}$, and let $T:=\max L$ and $B:=\min L$. Since $p_{0}$ is not pure, one has $T>B$. We contend that

$$
\lim _{n \rightarrow \infty} F^{n}\left(p_{0}\right)=\delta_{T}, \quad \lim _{n \rightarrow \infty} F^{-n}\left(p_{0}\right)=\delta_{B},
$$

where the convergence is in the sense of Hausdorff. Indeed, denoting $L^{\prime}=L \backslash\{T\}$, for all $a \in \prod_{i} A^{i}$ and each $p$ with the same support as $p_{0}$,

$$
\sum_{k \in L^{\prime}} p_{k} \mu_{k} \leq\left(1-p_{T}\right) \max _{k \in L^{\prime}} \mu_{k}
$$

and

$$
\sum_{k \in L} p_{k} \mu_{k}(a)<\mu_{T}(a)
$$

and hence

$$
\begin{aligned}
& \frac{p_{T} \mu_{T}(a)}{\sum_{k \in L} p_{k} \mu_{k}(a)}-p_{T}=\frac{p_{T}\left[\left(1-p_{T}\right) \mu_{T}(a)-\sum_{k \in L^{\prime}} p_{k} \mu_{k}\right]}{\sum_{k \in L} p_{k} \mu_{k}(a)} \\
& \geq \frac{p_{T}\left(1-p_{T}\right)\left(\mu_{T}-\max _{k \in L^{\prime}} \mu_{k}(a)\right)}{\sum_{k \in L} p_{k} \mu_{k}(a)} \\
&>p_{T}\left(1-p_{T}\right) \frac{\mu_{T}-\max _{k \in L^{\prime}} \mu_{k}(a)}{\mu_{T}(a)}>\delta p_{T}\left(1-p_{T}\right) .
\end{aligned}
$$

Similarly, one can show that if $L^{\prime \prime}=L \backslash\{B\}$, then

$$
p_{B}-\frac{p_{B} \mu_{B}}{\sum_{k \in L} p_{k} \mu_{k}} \geq p_{B}\left(1-p_{B}\right) \delta .
$$

Furthermore, for each pure state $\delta_{k}, F\left(\delta_{k}\right)=F^{-1}\left(\delta_{k}\right)=\left\{\delta_{k}\right\}$, where $F^{-1}(p)=\left\{p^{\prime} \mid p \in\right.$ $\left.F\left(p^{\prime}\right)\right\}$. Hence, for all $p$, $p^{\prime}$ with $p^{\prime} \in F(p),\left\|p^{\prime}-p\right\|_{\infty}<1$. Therefore, since $\operatorname{Gr}(F)$ is compact, we see that

$$
C:=\sup \left\{\left\|p^{\prime}-p\right\|_{\infty} \mid p^{\prime} \in F(p)\right\}<1 .
$$

Recall that $\mathcal{S}$ are the pure states in $\Delta(K)$. Denote $\varepsilon=\frac{1-C}{2}$ and define the set

$$
M:=\left\{p \in \Delta(K) \mid \bigwedge_{k \in K} p[k] \leq 1-\varepsilon\right\} .
$$

It then follows that the intersection of each $\mathcal{S}$-orbit with $M$ is non-empty and finite. (The non-emptiness follows by considering that any nontrivial orbit must contain elements arbitrarily close to at least two different vertices; since $C<1$ and by the choice of $\varepsilon$, the 
orbit cannot 'leapfrog' over $M$.) It follows from Proposition 2.5 that the $\mathcal{S}$-orbit equivalence relation is smooth. Hence, the auxiliary game possesses a stationary equilibrium by Theorem 5.3, and therefore, the original repeated game with symmetric incomplete information has an equilibrium strategy profile conditional only on the current belief over states.

\section{AN APPROXIMATION TECHNIQUE}

We present here an application of Theorem 3.1, which, in addition to being useful for its own sake, will be applied to deduce the general version of Theorem 8.2 below.

THEOREM 7.1: Let $\mathcal{E}$ be a smooth $C B E R$ on a Polish space $\Omega$, let $K$ be a compact metric space, and for each $n$, suppose $f_{n}: \Omega \rightarrow K$ is Borel. Then there exists a Borel mapping $g: \Omega \rightarrow K$ such that, for every class $C \in \Omega / \mathcal{E}$, the sequence of restrictions $\left(\left.f_{n}\right|_{C}\right)$ has a subsequence converging point-wise to $\left.g\right|_{C}$.

Note that, although Tychonoff's theorem guarantees a limit of $\left(f_{n}\right)$ in the Tychonoff topology on $\Omega^{K}$, the latter's topology does not have a countable basis and hence there need not (and generally will not) be a convergent subsequence of $\left(f_{n}\right)$. While it is true that applying Tychonoff's theorem on each class $C$ separately would give such a function $g$, doing so would not guarantee $g$ 's measurability.

PRoOF: We will apply Theorem 3.1, with parameter space $X=K$. Again, by Proposition 3.4, we can assume all classes of $\mathcal{E}$ are of the same cardinality as some $S$. Let $d: K \times K \rightarrow \mathbb{R}_{+}$be a metric on $K$. Fixing some positive weights $\left(\alpha_{s}\right)_{s \in S}$ with $\sum \alpha_{s}<\infty$ (e.g., $\alpha_{s}=2^{-s}$ if $S=\mathbb{N}$ ), we know that $K^{S}$ is compact (by Tychonoff's theorem), and metrizable with metric

$$
\rho\left(\left(x_{s}\right),\left(y_{s}\right)\right):=\sum_{s \in S} \alpha_{s} d\left(x_{s}, y_{s}\right) .
$$

The topology on $K^{S}$ is that of point-wise convergence.

Fix the language $\mathcal{L}=(=,+, \cdot,<, 0,1, d)$, where $d$ is a 2-place function symbol identified as the metric. ${ }^{26}$ Define the formula $\alpha$, with undetermined variables $\left(x_{s}\right)_{s \in S}$ and determined variables $\left(z_{s}^{n}\right)_{s \in S, n \in \mathbb{N}}{ }^{27}$

$$
\alpha\left(\left(x_{s}\right),\left(z_{s}^{n}\right)\right):=\bigwedge_{k \in \mathbb{N}} \bigvee_{n \in \mathbb{N}}\left(k \cdot \sum_{s \in S} \alpha_{s} d\left(z_{s}^{n}, x_{s}\right)<1\right),
$$

that is, $\forall k \in \mathbb{N}, \exists n \in \mathbb{N}, \rho\left(\left(z_{s}^{n}\right),\left(x_{s}\right)\right)<\frac{1}{k}$. Hence, $\alpha\left(\left(x_{s}\right),\left(z_{s}^{n}\right)\right)$ holds if and only if there is a subsequence $\left(z_{s}^{n_{k}}\right)$ of $\left(z_{s}^{n}\right)$ converging point-wise on $S$ to $\left(x_{s}\right)$. Let $\zeta_{n} \equiv f_{n}$. For any class $C$ of $\mathcal{E}$ and any bijection $\phi: C \rightarrow S$, the set $Z[\zeta, \phi]$ defined in Theorem 3.1 is just the set of limit points of $\left(f_{n}\left(\phi^{-1}(s)\right)_{s \in S}\right)_{n \in \mathbb{N}}$, which is, by Tychonoff's theorem, non-empty and compact. Hence, by applying Theorem 3.1(b), there exist $\Phi$ and $\Psi$ as described there. That is, $\Phi: \Omega \rightarrow S$ is Borel s.t. for each class $C$ of $\mathcal{E},\left.\Phi\right|_{C}$ is a bijection, and $\Psi(C)$ is a limit point in $K^{S}$ of $\left(f_{n}\left(\left.\Phi\right|_{C} ^{-1}(s)\right)_{s \in S}\right)_{n \in \mathbb{N}}$. Finally, set $g(\omega)=\Psi(\Phi(\omega))$.

Q.E.D.

\footnotetext{
${ }^{26}$ Formally, we should take the space $X=K \times \mathbb{R}$, identify $d(\cdot, \cdot)$ to be a metric on the product, and identify $K, \mathbb{R}$ with $K \times\left\{r_{0}\right\}$ and $\left\{k_{0}\right\} \times \mathbb{R}$ for some fixed $r_{0} \in \mathbb{R}, k_{0} \in K$.

${ }^{27}$ For this sentence, the weights $\left(\alpha_{s}\right)$ should be definable in the language $\mathcal{L}_{\omega_{1}, \omega}$; indeed, $\alpha_{s}=2^{-s}$ if $S=\mathbb{N}$ is suitable, as are $\alpha_{s}=\frac{1}{|S|}$ if $S$ is finite.
} 


\section{GRAPHICAL GAMES OF COUNTABLE DEGREE}

Let ( $\Omega, G$ ) be a graph (i.e., $G \subseteq \Omega \times \Omega$ is an irreflexive and symmetric relation). For finite graphs, Kearns, Littman, and Singh (2001) defined a graphical game to be a strategic form game such that $\Omega$ is the set of players and the payoff of each player depends only on his actions and those of his neighbors in $G$. Formally, suppose the (finite) action set of each agent is $A$. For a finite graph, letting $N(g)$ denote the set of the neighbors of $g$ in $\Omega$ under the vertex relation $G$, the payoff function of a player $g$ is a function $u_{g}$ : $A \times A^{N(g)} \rightarrow \mathbb{R}$. Payoffs extend multi-linearly in the usual way.

The generalization to infinite $G$ adds the requirement that the payoff function $u_{g}$ : $A \times A^{N(g)} \rightarrow \mathbb{R}$ is continuous in the Tychonoff topology. The existence of a Nash equilibrium was proven in Peleg (1969); an example showing the necessity of the continuity w.r.t. the Tychonoff topology is also presented. (Note that the continuity implies that $N(g)$ is countable for each $g \in G$.) However, even if $\Omega$ has a Borel structure and the payoffs obey some natural measurability requirements, the resulting equilibria need not be measurable; see Levy (2013a, Section 8).

Indeed, the model above generalizes immediately to the case in which $\Omega$ is a Polish space, $G \subseteq \Omega \times \Omega$ is Borel, the degree of $G$ is countable (i.e., for all $g \in G, N(g)$ is countable), and the payoffs are Borel. Specifically, the payoff function is a function $u$ : $\Omega \times A^{\Omega} \rightarrow \mathbb{R}$, understood as $u(g, a)$ being the payoff of $g$ when the profile $a$ is played, such that $u(g, \cdot)$ depends only on the actions of $g$ and his neighbors. (Formally, if $g \in \Omega$ and $a, a^{\prime}: \Omega \rightarrow A$ are two action profiles such that $a(g)=a^{\prime}(g)$, and $a(h)=a^{\prime}(h)$ for each $h \in N(g)$, then $u(g, a)=u\left(g, a^{\prime}\right)$.)

Given these definitions, we will assume that:

- $u_{g}$ is continuous in the Tychonoff topology on $A \times A^{N(g)}$.

- Whenever $\alpha: \Omega \times \Omega \rightarrow A$ is Borel, $g \rightarrow u(g, \alpha(g, \cdot))$ is Borel.

DEFINITION 8.1: For a graphical game $(\Omega, G)$ over a Polish space with a Borel edge relation $\mathcal{E}$, the equivalence relation induced by the edges $G$ (i.e., the symmetric, reflexive, transitive closure of $\mathcal{E}$ ) straightforwardly satisfies the conditions for being a CBER. We will call such a game a purely atomic graphical game.

A (measurable) Nash equilibrium of a graphical game is naturally a (measurable) mapping $\sigma: G \rightarrow \Delta(A)$ such that $u(g, \sigma) \geq u\left(g,\left(b,(\sigma(h))_{h \neq g}\right)\right)$ for each $g \in G$ and each $b \in A$, that is, replacing the mixed action chosen by player $g$ by any pure action $b$ (while the mixed actions of the other players remain fixed) does not increase $g$ 's payoff.

THEOREM 8.2: If the equivalence relation $\mathcal{E}$ of a purely atomic graphical game is smooth, then the game admits a measurable Nash equilibrium.

PROOF: We begin with the case in which each vertex has only finitely many graph neighbors. Given the similarity to the proofs of Theorems 5.2 and 4.7, we need only sketch a proof. Each connected component of $G$-that is, each class of $\mathcal{E}$-is countable and by assumption smooth, and hence an equilibrium exists for each component. The existence of an equilibrium over the entire graph is easily expressed using a quantifier-free formula (i.e., only countable quantifiers) in $\mathcal{L}_{\omega_{1}, \omega}$ of an appropriate language $\mathcal{L}{ }^{28}$ Our assump-

\footnotetext{
${ }^{28}$ This is where we need the assumption that each vertex has finitely many neighbors, since otherwise the payoff function can take on a different value for each of the uncountably many profiles of actions that can be played; the logical language we have been using cannot accommodate uncountably many free variables.
} 
tions imply that the notion of 'neighbor' and the payoffs are Borel; these will constitute, in some form, the functions $\zeta_{j}$ in Theorem 3.1. The result then follows from Theorem 3.1.

We now deduce from this the existence of a measurable equilibrium in the case in which vertexes may have countably infinitely many neighbors. Fix an increasing sequence of Borel subsets of $\Omega, \Omega_{1} \subseteq \Omega_{2} \subseteq \Omega_{3} \subseteq \ldots$ with $\bigcup_{n} \Omega_{n}=\Omega$, such that for each $n$ and each equivalence class $C$ of $\mathcal{E}, \Omega_{n} \cap C$ is non-empty and finite; such is seen to exist by applying Proposition 2.6, with $S=\left\{s_{1}, s_{2}, s_{3}, \ldots\right\}$, and setting $\Omega_{n}=\Phi^{-1}\left(\left\{s_{1}, \ldots, s_{n}\right\}\right)$.

Fix some $a_{0} \in A$. Recall that $u: \Omega \times A^{\Omega} \rightarrow \mathbb{R}$, understood as $u(g, a)$ being the payoff of $g$ when the profile $a$ is played. For an action profile $a$ and element $h \in \Omega$, denote by $a_{h}$ the action in $a$ associated with the index $h$. Then, for each $n \in \mathbb{N}$, denote

$$
u_{n}(g, a):=u\left(g, \tilde{a}^{n}\right),
$$

where

$$
\tilde{a}_{h}^{n}= \begin{cases}a_{h} & \text { if } h \in \Omega_{n}, \\ a_{0} & \text { if } h \notin \Omega_{n} .\end{cases}
$$

$u_{n}$ then satisfies the required measurability and continuity conditions, and each player's payoff under $u_{n}$ depends only on his action and the actions of neighbors in $\Omega_{n}$.

For each $n$, let $\sigma^{n}$ be an equilibrium of the game restricted to $\Omega_{n}$, with payoff function $u_{n}$. For each fixed equivalence class $C$ and $g \in C$, the payoff functions $u(g, \cdot)$ and $u_{n}(g, \cdot)$ are well-defined functions on $A^{C}$. It follows from the continuity of $u(g, \cdot)$ that, for all $g \in G, u_{n}(g, \cdot) \rightarrow u(g, \cdot)$ uniformly on $A^{C}$, and since $A^{C}$ is compact, $u(g, \cdot)$ is uniformly continuous on $A^{C}$. From this, it follows that any limit point ${ }^{29}$ of $\left.\tilde{\sigma}^{n}\right|_{C}$ in $(\Delta(A))^{C}$ is an equilibrium of $u$ over $C$. Hence, applying Theorem 7.1 yields a Borel equilibrium $\sigma$ on $\Omega$.

Q.E.D.

\section{BETTING}

\subsection{Preliminaries}

If $(\Omega, \mathcal{B})$ is a measurable space, $\mu \in \Delta(\Omega)$, and $\mathcal{F}$ is a sub- $\sigma$-algebra of $\mathcal{B}$, then (see Blackwell and Ryll-Nardzewski (1963)) a proper regular conditional distribution (henceforth, proper RCD) of $\mu$, given $\mathcal{F}$, is a mapping $t: \Omega \times \mathcal{B} \rightarrow[0,1]$ such that for each $B \in \mathcal{B}, \omega \rightarrow t_{\omega}(B)$ is Borel and measurable in $\mathcal{F}$, and such that

$$
\mu(B)=\int_{\Omega} t_{\omega}(B) d \mu(\omega) \quad \text { for all } B \in \mathcal{B},
$$

and

$$
t_{\omega}(A)=1 \quad \text { for } \mu \text {-a.e. } \omega \in A \in \mathcal{F} .
$$

It can be shown that Equation (9.1) implies that, for every $T \in \mathcal{B}$,

$$
t_{\omega}(T)=E_{\mu}\left[1_{T} \mid \mathcal{F}\right](\omega), \quad \mu \text {-a.e. } \omega \in \Omega .
$$

In terms that may be more familiar for game theorists, a proper RCD $t$ of a probability measure $\mu$ may be thought of as the posterior $t$ of a prior $\mu$ with respect to a knowledge structure $\mathcal{F}=\sigma(\mathcal{E})$, where $\sigma\left(\mathcal{E}^{i}\right)$ is the $\sigma$-algebra of Borel $\mathcal{E}^{i}$-saturated sets.

\footnotetext{
${ }^{29} \tilde{\sigma}^{n}$ extends $\sigma^{n}$ to $a_{0}$ outside of $\Omega_{n}$.
} 
Given a type space $\left(\Omega, \mathcal{B},\left(t^{i}\right)_{i \in I}\right)$, a measure $\mu^{i} \in \Delta(\Omega)$ such that $t^{i}$ is a proper RCD for $\mu^{i}$ given $\sigma\left(\mathcal{E}^{i}\right)$ is a prior for $t^{i}$. A common prior is a measure $\mu$ that is a prior for the type functions of all the players $i \in I$.

\subsection{Acceptable Bets}

For a type space $\tau=\left(t_{i}\right)_{i \in I}$ on a space $\Omega$, a bet is a list of $\left(f^{i}\right)_{i \in I}$ of bounded ${ }^{30}$ random variables $f^{i}: \Omega \rightarrow \mathbb{R}$ such that $\sum_{i \in I} f^{i}(\omega)=0$ for all $\omega \in \Omega$. An acceptable bet is a bet that satisfies the condition that

$$
E^{i}\left[f^{i} \mid \omega\right]=\int_{\Omega} f^{i}(s) d t_{\omega}^{i}[s]>0 \quad \text { for all } i \in I, \omega \in \Omega .
$$

Note that when the underlying space is discrete (e.g., if it is a countable component of a purely atomic type space), there are no measurability requirements on the bet.

THEOREM 9.1: If the common knowledge equivalence relation of a purely atomic type space is smooth, and there is an acceptable bet on each common knowledge component, then there is an analytically measurable acceptable bet on the entire space.

PROOF: Fix a space $S$ such that all common knowledge classes are of cardinality $S$; by Proposition 3.4, it suffices to deal with the case in which all classes are of equal cardinality. We again define a quantifier-free formula in the language $\mathcal{L}_{\omega_{1}, \omega}$, for $\mathcal{L}=(+,=,>)$, where again the indices $s, t$ are understood to be in $S$, and $i, j$ are indices of players in $I$.

- The relation $\sum(\cdot)>0$, where $\sum_{s \in S} x_{s}>0$ expresses-similarly to Theorem 4.7-the summability of a series and the positivity of the sum; we write this for clarity instead of a more formal formula $\gamma\left(\left(x_{s}\right)_{s \in S}\right)$.

- Let

$$
\alpha\left(\left(\tau_{j, s, t}\right),\left(x_{j, s}\right)\right)=\bigwedge\left(\sum_{j} x_{j, u}=0\right) \bigwedge_{u} \bigwedge_{j}\left(\sum_{v} \tau_{j, u, v} x_{j, v}>0\right),
$$

which expresses the fact that if $\tau_{j, s, t}$ is the probability that player $j$ associates to state $t$ when in state $s$, then $\left(x_{j, s}\right)$ is an acceptable bet.

The proof then follows the similar template, along with our assumption that every component possesses an acceptable bet, with the notable exception that we must use Theorem 3.1(a) instead of Theorem 3.1(b) since the collection of acceptable bets need not be closed. ${ }^{31}$

Q.E.D.

Hellman and Levy (2017) presented an example, adapted from one in Lehrer and Samet (2011), of a hyperfinite but not smooth knowledge space in which there is no measurable acceptable bet on the entire space but one can construct an acceptable bet individually over each common knowledge component. Hence, smoothness is necessary in the statement of Theorem 9.1.

\footnotetext{
${ }^{30}$ We assume boundedness to avoid anomalies; see Feinberg (2000) and Hellman (2014a).

${ }^{31}$ It is not $\sigma$-compact in general either, which follows from the fact that the positive sequences $(0, \infty)^{\mathbb{N}}$ is not $\sigma$-compact. This clearly is equivalent to $\mathbb{R}^{\mathbb{N}}$ not being $\sigma$-compact, and indeed it follows from the Baire category theorem that any completely metrizable topological vector space is not $\sigma$-compact.
} 
That same example also exhibits the anomaly of a knowledge space with a common prior which nevertheless admits an acceptable bet over each common knowledge component, a striking situation in light of the Aumann No Disagreement Theorem. Theorem 9.2 shows that smoothness guarantees that such an anomaly cannot occur. This result was also proved in Hellman and Levy (2017) in a different way. ${ }^{32}$

THEOREM 9.2: On a smooth type space with a common prior $\mu$, there is no acceptable bet for $\mu$-almost every common knowledge component. ${ }^{33}$

PRoof: Suppose by way of contradiction that there is a subset $\Theta \subseteq \Omega$ that satisfies the properties that it is common knowledge for all the players, that $\mu(\Theta)>0$, and that over every common knowledge component in $\Theta$ there is an acceptable bet. By Theorem 9.1 restricted to $\Theta$, one can then construct an analytically measurable acceptable bet over all of $\Theta$. However, $\Theta$ admits a common prior probability measure restricted to $\Theta$, namely $\mu(\cdot) / \mu(\Theta)$. The existence of a common prior on a space excludes the existence of an acceptable bet over the space. This is a contradiction.

Q.E.D.

\section{CONCLUSION AND DIRECTIONS FOR FURTHER RESEARCH}

We have shown how the measurable selection theorem (Theorem 3.1) can be used to establish the existence of measurable equilibria in smooth purely atomic Bayesian, stochastic, and graphical games. What other types of games are amenable to being studied under the purely atomic paradigm?

Furthermore, all the focus in this paper is on purely atomic games and countable Borel equivalence relations. What can be said about equilibrium existence in games that are not purely atomic, for example, in Bayesian games in which the knowledge structures of some players admit knowledge partitions with uncountably many states, or in stochastic games with uncountable orbits? $?^{34}$

\section{APPENDIX A: A CONDITION FOR SMOOTHNESS}

For practical purposes, and to aid the reader in grasping the concept of smoothness, we present Theorem A.1, which yields a prototypical but hardly exhaustive class of smooth relations. Intuitively, this theorem states that as long as, within each equivalence class, the elements of the class do not get 'bunched up', the equivalence relation is smooth. A particular case of this occurs if, for some metric $d$, the condition $\inf _{x \neq y, x, y \in C} d(x, y)>0$ holds for each class $C$ of $\mathcal{E}$; one may think of this as requiring that the elements within each class 'keep their distance', as witnessed by inf $d(x, y)$.

\footnotetext{
${ }^{32}$ It was shown there that under smoothness and a common prior, almost every common knowledge component has a common prior. Hence, almost every common knowledge component cannot possess an acceptable bet.

${ }^{33}$ That is, the union of the collection of common knowledge components on which an acceptable bet may exist is of $\mu$-measure 0 .

${ }^{34}$ With respect to the existence of measurable Bayesian equilibria in games with continuum-many types, Milgrom and Weber (1985) proved that such equilibria exist when players have absolutely continuous information. Games with absolutely continuous information form a disjoint class from the class of purely atomic Bayesian games. More details on this appear in Hellman and Levy (2017), based on Stinchcombe (2011). We are not aware of similar results for stochastic games.
} 
THEOREM A.1: Suppose $\Omega$ is a Polish space and that $\mathcal{E}$ is a CBER such that for each equivalence class $C$ of $\mathcal{E}$ and each $z \in C, z$ is an isolated point of $C$, i.e., $z \notin \overline{C \backslash\{z\}}$. Then $\mathcal{E}$ is smooth.

REMARK A.2: Theorem A.1 complements, to some extent, Sullivan, Weiss, and Wright (1986, Lemma 1.1), which shows that for relations induced by a countable group of homeomorphisms of Polish spaces, the existence of dense equivalence class implies nonsmoothness. Hence, although Theorem A.1 is not an exhaustive criterion for smoothness, it well encompasses its spirit.

PRoOF: Let $\left(V_{n}\right)_{n \in \mathbb{N}}$ be a countable basis for $\Omega$. For each $n \in \mathbb{N}$, define $\psi_{n}: \mathbb{N} \rightarrow$ $\{0,1,2, \ldots, \infty\}$ by

$$
\psi_{n}(\omega)=\left|\left\{V_{n} \cap[\omega]_{\mathcal{E}}\right\}\right| .
$$

Each $\psi_{n}$ is measurable, by Proposition 2.6. Our assumption that, for each equivalence class $C$ of $\mathcal{E}$, each point is an isolated point of $C$, further implies that $M(\omega)=\{n \in \mathbb{N} \mid$ $\left.\psi_{n}(\omega)=1\right\}$ is non-empty for each $\omega$; indeed, for each $z \in C$, there exists $n$ such that $V_{n} \cap C=\{z\}$, and such that the function $m(\cdot)=\min E(\cdot)$ is measurable.

Hence, by construction, the Borel set

$$
\mathcal{E} \cap\left\{(\omega, \theta) \in \Omega \times \Omega \mid \theta \in V_{m(\omega)}\right\}
$$

is the graph of a Borel function which is constant on each atom. It follows that $\mathcal{E}$ is smooth. Q.E.D.

\section{APPENDIX B: FIRST-ORDER AND INFINITARY LOGIC}

\section{B.1. First-Order Logic}

We begin by recalling the standard notions of first-order logic; see, for example, Enderton (2001). Let $\mathcal{L}$ be a finite base language, that is, a fixed first-order language, consisting of:

- The connective symbols ${ }^{35} \rightarrow, \neg, \wedge, \vee$, and the equality symbol $=$.

- A finite or countable collection of variables $V$.

- The quantifier symbols $\forall, \exists$.

- A (possibly empty) collection of constant symbols, denoted $\mathcal{C}$.

- For each $n$, some possibly empty collection $\mathcal{R}_{n}$ of $n$-place predicate symbols; denote $\mathcal{R}=\bigcup_{n=1}^{\infty} \mathcal{R}_{n}$. (Equality is considered a 2-place predicate symbol as well.)

- For each $n$, some possibly empty collection $\mathcal{F}_{n}$ of $n$-place function symbols; denote $\mathcal{F}=\bigcup_{n=1}^{\infty} \mathcal{F}_{n}$.

The collection of formulae is constructed inductively in the well-known manner:

- The terms consist of those expressions created by the variables, the constant symbols, and the function symbols by repeated composition.

- The atomic formulae are of the form $R\left(t_{1}, \ldots, t_{n}\right)$ for some predicate symbol $R$ and terms $t_{1}, \ldots, t_{n}$.

- The well-formed formulae are those expressions built up from the atomic formulas and repeated use of the connective symbols and the quantifier symbols.

\footnotetext{
${ }^{35}$ Actually, at a minimum $\rightarrow$ and $\neg$ alone can suffice.
} 
The free variables of an atomic formula are simply those variables that appear in it. If $F_{\alpha}, F_{\beta}$ are the free variables of formulas $\alpha, \beta$, respectively, and $v$ is a variable, then $F_{\alpha}$ (resp. $F_{\alpha} \cup F_{\beta}$, resp. $F \backslash\{v\}$ ) are the free variables of $\neg \alpha$ (resp. $\alpha \rightarrow \beta, \alpha \wedge \beta$, and $\alpha \vee \beta$, resp. $\forall v \alpha$ and $\exists v \alpha$ ), respectively. Variables that are not free are called bound variables. A formula with no free variables is called a sentence.

A structure $\mathcal{M}$ consists of a set that is called a universe (by mildly abusive notation denoted also $\mathcal{M}$ ), and assignments of a set $R_{\mathcal{M}} \subseteq \mathcal{M}^{n}$ to each $n$-place predicate, an element $c_{\mathcal{M}} \in \mathcal{M}$ to each constant symbol $c \in \mathcal{C}$, and a function $f_{\mathcal{M}}: \mathcal{M}^{n} \rightarrow \mathcal{M}$ to each $n$-place function symbol. For elements $m_{1}, \ldots, m_{n}, R\left(m_{1}, \ldots, m_{n}\right)$ holds if $\left(m_{1}, \ldots, m_{n}\right) \in R_{\mathcal{M}}$; in the usual inductive and intuitive way, for any well-formed formula $\alpha$ with free variables $x_{1}, \ldots, x_{n}$, it is well defined for each $m_{1}, \ldots, m_{n}$ whether $\alpha\left(m_{1}, \ldots, m_{n}\right)$ holds or not. If $\Gamma$ is a collection of sentences such that every sentence in $\Gamma$ is true in the structure $\mathcal{M}$, we say that $\mathcal{M}$ is a model for $\Gamma$.

\section{B.2. Infinitary Logic}

First-order logic, for all its expressiveness, can sometimes fail to enable the expression of some of the most elementary expressions used in everyday discussions of mathematics. A simple but striking example involves the formal expression of finiteness. One can state that there are 5 elements, or $10^{28}$ elements in a model perfectly well in first-order logic, but there is simply no way to say 'there are $n$ elements, where $n$ is any finite integer' by way of a first-order sentence, despite the ease with which we use such expressions in informal daily language. Indeed, it is a well-known corollary of the compactness theorem (e.g., Enderton (2001)) that, in first-order logic, there is no sentence $\phi$ that witnesses the finiteness of a model; that is, there exists no sentence $\phi$ such that $\mathcal{M}$ is a model for $\phi$ if and only if the universe of $\mathcal{M}$ is finite.

Therefore, we now extend this language to an infinitary language; see, for example, Keisler (1971). For clarity, we adopt only the generalization we will need in this paper, that of the language $\mathcal{L}_{\omega_{1}, \omega}$. In this language, in addition to the formulae allowed above, we allow formulae of the form $\bigwedge_{n=1}^{\infty} \alpha_{n}$ and $\bigvee_{n=1}^{\infty} \alpha_{n}$, whenever $\left(\alpha_{n}\right)_{n=1}^{\infty}$ is a countable collection of formulae. If $F_{n}$ is the collection of free variables in $\alpha_{n}$, then $\bigcup_{n} F_{n}$ is the collection of free variables in $\bigwedge_{n=1}^{\infty} \alpha_{n}$ and $\bigvee_{n=1}^{\infty} \alpha_{n}$. In such a case, given a structure $\mathcal{M}$, if $F=\bigcup_{n} F_{n}=\left(x_{j}\right)_{j \in F}$ and $\left(m_{j}\right)_{j \in F}$ is a collection in $\mathcal{M}$, then $\left(\bigvee_{n=1}^{\infty} \alpha_{n}\right)\left(\left(m_{j}\right)_{j \in F}\right)$ (resp. $\left.\left(\bigwedge_{n=1}^{\infty} \alpha_{n}\right)\left(\left(m_{j}\right)_{j \in F}\right)\right)$ holds if and only if $\alpha_{n}\left(\left(m_{j}\right)_{j \in F_{n}}\right)$ holds for some (resp. all) $n$.

EXAMPLE B.1: It is a well-known corollary of the compactness theorem (e.g., Enderton (2001)) that in the standard first-order language, there is no sentence $\phi$ that witnesses the finiteness of a model; that is, there exists no sentence $\phi$ such that $\mathcal{M}$ is a model for $\phi$ if and only if the universe of $\mathcal{M}$ is finite. There is, however, such a sentence $\phi$ in the infinitary logic $\mathcal{L}_{\omega_{1}, \omega}$.

For each $n \in \mathbb{N}$, let $\phi_{n}$ be the sentence

$$
\phi_{n}=\exists x_{1} \cdots \exists x_{n} \forall y\left(y=x_{1}\right) \vee \cdots \vee\left(y=x_{n}\right) .
$$

$\phi_{n}$ states that there are at most $n$ elements. Then, let

$$
\phi=\bigvee_{n=1}^{\infty} \phi_{n} .
$$

Clearly, $\mathcal{M}$ is a model for $\phi$ if and only if there is $n$ such that $\mathcal{M}$ has at most $n$ elements, that is, if and only if $\mathcal{M}$ is finite. 
EXAMPLE B.2: Suppose we have the language $\mathcal{L}=(+,>, 0)$. Let $\mathbb{R}$ be a structure in which the universe consists of the real numbers and these symbols have the standard interpretation. We can write a formula $\phi$ with countably many free variables $x_{1}, x_{2}, \ldots$ such that, for any $m_{1}, m_{2}, \ldots$ in $\mathbb{R}, \phi\left(m_{1}, m_{2}, m_{3}, \ldots\right)$ holds if and only if $\lim _{n \rightarrow \infty} m_{n}$ exists (in the finite sense). Indeed, write, for each $n \in \mathbb{N}$,

$$
\alpha_{n}(x, L, \varepsilon)=(L+\varepsilon>x) \wedge(x+\varepsilon>L),
$$

and

$$
\beta_{n}\left(L, \varepsilon, x_{1}, x_{2}, \ldots\right)=\bigwedge_{m \geq n} \alpha_{n}\left(x_{m}, L, \varepsilon\right),
$$

and finally

$$
\phi\left(x_{1}, x_{2}, \ldots\right)=\exists L \forall \varepsilon\left((\varepsilon>0) \rightarrow\left(\bigvee_{n=1}^{\infty} \beta_{n}\left(L, \varepsilon, x_{1}, x_{2}, \ldots\right)\right)\right)
$$

Obviously, in the standard first-order language over $\mathcal{L}$, such a statement cannot be encoded, since only finitely many variables may appear in the formula.

Of particular interest for us will be the quantifier-free formulae, that is, those formulae which are constructed without the use of quantifiers.

EXAMPLE B.3: Let us re-do Example B.2 in a quantifier-free way, with the larger language $\mathcal{L}=(+, \cdot,>, 0,1)$. Since we do not want to state the existence of the limit explicitly in the sentence (something that would involve a quantifier), we use the Cauchy criterion. Let

$$
\gamma_{n}(x, y)=(n \cdot y+1>n \cdot x) \wedge(n \cdot y<n \cdot x+1),
$$

(i.e., $\left.|x-y|<\frac{1}{n}\right)$ where $n$ is shorthand for $(1+\cdots+1) n$ times, and then

$$
\psi\left(x_{1}, x_{2}, \ldots\right)=\bigwedge_{K \in \mathbb{N}} \bigvee_{N \in \mathbb{N}} \bigwedge_{n, m \geq N} \gamma_{K}\left(x_{n}, x_{m}\right),
$$

that is, $\forall K \in \mathbb{N}, \exists N \in \mathbb{N}, \forall_{m, n \geq N},\left|x_{m}-x_{n}\right|<\frac{1}{K}$.

\section{APPENDIX C: PROOF OF THEOREM 3.1}

(a) Let $\operatorname{Sym}(S)$ denote the symmetric group on $S$, that is, the set of bijections from $S$ to $S$, endowed with the Tychonoff topology as a subspace of $S^{S}$, making it a Polish space; see, for example, Becker and Kechris (1996, Section 1.5). Begin with any Borel map $\hat{\Phi}: \Omega \rightarrow S$ for which $\left.\hat{\Phi}\right|_{C}: C \rightarrow S$ is bijective for each class $C$; such a map exists by Proposition 2.6. Define a correspondence $\Lambda: \Omega / \mathcal{E} \rightarrow \operatorname{Sym}(S) \times X^{F_{U}}$ by

$$
\Lambda(C):=\left\{\left(\sigma, x^{F_{U}}\right) \in \operatorname{Sym}(S) \times X^{F_{U}} \mid x^{F_{U}} \in Z\left[\zeta,\left.\sigma \circ \hat{\Phi}\right|_{C}\right]\right\} .
$$

By assumption, for all $C \in \Omega / \mathcal{E}, \Lambda(C) \neq \emptyset$. This correspondence $\Lambda$ has a Borel graph; indeed, define two Borel subsets of $\frac{\Omega}{\mathcal{E}} \times \operatorname{Sym}(S) \times X^{F_{D}} \times X^{F_{U}}$ by

$$
W=\left\{\left(C, \sigma, \zeta\left[\left.\sigma \circ \hat{\Phi}\right|_{C}\right], z\right) \mid C \in \frac{\Omega}{\mathcal{E}}, \sigma \in \operatorname{Sym}(S), z \in X^{F_{U}}\right\},
$$


and

$$
V=\Omega / \mathcal{E} \times \operatorname{Sym}(S) \times Z .
$$

$V$ is clearly Borel; to show that $W$ is Borel, note that we can write $\zeta\left[\left.\sigma \circ \hat{\Phi}\right|_{C}\right]=\sigma_{*}\left(\zeta\left[\left.\hat{\Phi}\right|_{C}\right]\right)$, where $\sigma_{*}(x)_{j}\left(s_{1}, \ldots, s_{N_{j}}\right)=x_{j}\left(\sigma^{-1}\left(s_{1}\right), \ldots, \sigma^{-1}\left(s_{N_{j}}\right)\right)$ for $x \in X^{F_{D}}$. The mapping $\frac{\Omega}{\mathcal{E}} \rightarrow$ $X^{F_{D}}$ given by $C \rightarrow \zeta\left[\left.\hat{\Phi}\right|_{C}\right]$ is shown to be Borel by Proposition 2.6.

Then $\operatorname{Gr}(\Lambda)$ is the projection of $W \cap V$ to $\frac{\Omega}{\varepsilon} \times \operatorname{Sym}(S) \times X^{F_{U}}$, and this projection is injective on $W$. Hence, by the Aumann Selection Theorem (see the version of, e.g., Wagner (1977, Corollary 5.2) or Kechris (1995, Theorem 18.1)), there exists an analytically measurable selector $(\hat{\sigma}, \Psi)$ of $\hat{S}$. Define $\Phi: \Omega \rightarrow S$ by

$$
\Phi(\omega):=\hat{\sigma}(\iota(\omega)) \circ \hat{\Phi}(\omega),
$$

where $\iota: \Omega \rightarrow \Omega / \mathcal{E}$ is the quotient map; $\Phi, \Psi$ is then the desired pair.

(b) In this case, take $\Phi=\hat{\Phi}$ and define the simpler correspondence $\Lambda: \Omega / \mathcal{E} \rightarrow \Omega^{F_{U}}$ by

$$
\Lambda(C):=\left\{x^{F_{U}} \in X^{F_{U}} \mid x^{F_{U}} \in Z\left[\zeta,\left.\Phi\right|_{C}\right]\right\} .
$$

$\Lambda$ is now a correspondence with non-empty $\sigma$-compact values, and a Borel graph, which has a Borel-measurable selection $\Psi$ by the Arsenin-Kunugui theorem (see, e.g., Kechris (1995, Theorem 35.46)).

\section{APPENDIX D: PROOF OF THEOREM 5.3}

We may assume that the system $\mathcal{S}$ is closed under finite unions and that $\Omega \in \mathcal{S}$; this completion of the system preserves $\mathcal{S}$-orbits. (Note that if $S \in \mathcal{S}$ can be written as a union of strict subsets in $\mathcal{S}$, then there are no $S$-orbits as $\hat{S}=\emptyset$.) By assumption, each $S \in \mathcal{S}$ is $Q$-invariant, hence we may consider the stochastic game restricted to states in $S$, denoting this game by $\Gamma_{S}$.

We construct equilibria on $\left(\Gamma_{S}\right)_{S \in \mathcal{S}}$ in such a way that if $\sigma_{S}, \sigma_{T}$ have been constructed for $S, T \in \mathcal{S}$, then they agree on $S \cap T \in \mathcal{S}$ (and, hence, we have defined an equilibrium $\sigma_{S \cup T}$ on $\Gamma_{S \cup T}$ ). For each minimal set $S \in \mathcal{S}$, the orbits of $\Gamma_{S}$ are countable, hence an equilibrium $\sigma_{S}$ exists on $\Gamma_{S}$ by Theorem 5.2.

From here, continue inductively: fix $S \in \mathcal{S}$ such that the equilibrium has not yet been constructed but has been constructed for the unique maximal subset $T \in \mathcal{S}$ of $S .{ }^{36}$ Denote $\hat{S}:=S \backslash T$. Define an auxiliary game, with the same sets of players and actions, by (see Figure 3):

- $\Omega^{\prime}=\hat{S} \cup(\hat{S} \times T) \cup\{\emptyset\}$.

- Payoffs $r^{\prime}$, for all $a \in \prod_{i} A^{i}$, given by

$$
\begin{aligned}
r^{\prime}(s, a) & =r(s, a), \quad \forall s \in \hat{S}, \\
r^{\prime}((s, t), a) & =\gamma_{\sigma_{T}}^{\lambda}(t), \quad \forall s \in \hat{S}, \forall t \in T,
\end{aligned}
$$

where $\gamma$ was defined in (5.1), and

$$
r^{\prime}(\emptyset, a)=0
$$

\footnotetext{
${ }^{36}$ Such a unique maximal subset of $S$ in $\mathcal{S}$ exists as we have assumed that $\mathcal{S}$ is finite and closed under finite union and intersection.
} 


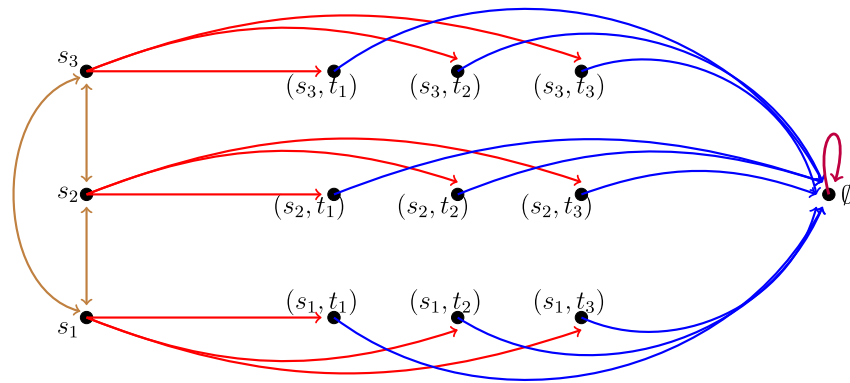

States in $S^{\prime}$ : Payoffs: As in $\Gamma$ Transition: Within $S^{\prime}$ or to $S^{\prime} \times T$

$$
\begin{gathered}
\text { States in } S^{\prime} \times T \text { : } \\
\text { Payoffs: } \gamma_{\sigma_{T}}^{\lambda} \\
\text { Transition: To } \emptyset
\end{gathered}
$$

$\emptyset$ is Absorbing Payoffs: 0

FIGURE 3.-Transitions in the auxiliary game in proof of Theorem 5.3.

- Transitions $q^{\prime}$ given, for all $a \in \prod_{i} A^{i}$ and for all $s, s^{\prime} \in \hat{S}$ with $s \neq s^{\prime}$ and $t \in T$, by

$$
q^{\prime}(z \mid s, a)= \begin{cases}q(z \mid s, a) & \text { if } z \in \hat{S} \\ q(t \mid s, a) & \text { if } z=(s, t) \\ 0 & \text { if } z=\left(s^{\prime}, t\right) \text { or if } z=\emptyset\end{cases}
$$

and

$$
q(\emptyset \mid(s, t), a)=q(\emptyset \mid \emptyset, a)=1 .
$$

This auxiliary game has countable orbits-every orbit of the auxiliary game is contained in a set of the form $C \cup(C \times Q(C)) \cup\{\emptyset\}$, where $C$ is an $S$-orbit of the original game-and hence an equilibrium $\sigma^{\prime}$ exists by Theorem 5.2. Now, if we define

$$
\sigma_{S}(s)= \begin{cases}\sigma_{T}(s) & \text { if } s \in T, \\ \sigma^{\prime}(s) & \text { if } s \in \hat{S}=S \backslash T,\end{cases}
$$

then $\sigma_{S}$ is an equilibrium of $\Gamma_{S}$, which is what we were seeking.

Continuing in this way, we eventually construct a measurable stationary equilibrium of $\Gamma_{\Omega}$, which is the original stochastic game with which we began.

\section{REFERENCES}

Becker, H., AND A. Kechris (1996): The Descriptive Set Theory of Polish Group Actions. Cambridge Texts in the History of Philosophy. Cambridge: Cambridge University Press. [625]

Bertsekas, D., And S. SHREve (1996): Stochastic Optimal Control: The Discrete Time Case. Belmont, MA: Athena Scientific. [611]

Blackwell, D., AND C. RYll-NARDZEWSKI (1963): "Non-existence of Everywhere Proper Conditional Distributions," Annals of Mathematical Statistics, 34 (1), 223-225. [620]

Brandenburger, A., AND E. Dekel (1987): “Common Knowledge With Probability 1,” Journal of Mathematical Economics, 16 (3), 237-245. [606]

DougherTy, R., S. JACKSON, AND A. S. KECHRIS (1994): “The Structure of Hyperfinite Borel Equivalence Relations," Transactions of the American Mathematical Society, 341 (1), 193-225. [599] 
EnderTON, H. (2001): A Mathematical Introduction to Logic. Burlington, MA: Harcourt/Academic Press. [596, 623,624]

FEINBERG, Y. (2000): “Characterizing Common Priors in the Form of Posteriors," Journal of Economic Theory, 91 (2), 127-179. [621]

Feldman, J., AND C. C. Moore (1975): "Ergodic Equivalence Relations, Cohomology, and von Neumann Algebras," Bulletin of the American Mathematical Society, 81 (5), 921-924. [602]

FORGES, F. (1982): "Infinitely Repeated Games of Incomplete Information: Symmetric Case With Random Signals," International Journal of Game Theory, 11 (3), 203-213. [615]

HARSANYI, J. C. (1967): "Games With Incomplete Information Played by Bayesian Players, I-III Part I. The Basic Model," Management Science, 14 (3), 159-182. [594]

Hellman, Z. (2014a): “Countable Spaces and Common Priors," International Journal of Game Theory, 43 (1), 193-213. [621]

(2014b): "A Game With No Bayesian Approximate Equilibria," Journal of Economic Theory, 153, 138-151. [594,606-608]

Hellman, Z., AND Y. J. LeVY (2017): "Bayesian Games With a Continuum of States,” Theoretical Economics, 12, 1089-1120. [594,595,606,608,621,622]

(2019): "Bayesian Updating Induced Non-smooth Relations" (in preparation). [595,616]

Kearns, M. J., M. L. LitTMAN, AND S. P. Singh (2001): "Graphical Models for Game Theory," 253-260. [619]

KeChris, A. (1995): Classical Descriptive Set Theory. Graduate Texts in Mathematics. New York: Springer Verlag. [598,599,602,626]

KeChris, A., AND B. Miller (2004): Topics in Orbit Equivalence. Lecture Notes in Mathematics. Berlin, Heidelberg: Springer Verlag. [598,599]

KeISLER, H. (1971): Model Theory for Infinitary Logic: Logic With Countable Conjunctions and Finite Quantifiers. Studies in Logic and the Foundations of Mathematics. Amsterdam: North-Holland Publishing Company. [597,624]

KOHLBERG, E., AND S. ZAMIR (1974): "Repeated Games of Incomplete Information: The Symmetric Case," Annals of Statistics, 2 (5), 1040-1041. [615]

LEHRER, E., AND D. SAMET (2011): “Agreeing to Agree,” Theoretical Economics, 6 (2), 269-287. [594,606,607, 621]

LEVY, Y. (2013a): "A Cantor Set of Games With No Shift-Homogeneous Equilibrium Selection," Mathematics of Operations Research, 38 (3), 492-503. [594,619]

(2013b): "Discounted Stochastic Games With No Stationary Nash Equilibrium: Two Examples," Econometrica, 81 (5), 1973-2007. [593,612]

LEVY, Y. J., AND A. MCLENNAN (2015): "Corrigendum to 'Discounted Stochastic Games With No Stationary Nash Equilibrium: Two Examples',” Econometrica, 83 (3), 1237-1252. [593,612]

MERTENS, J., S. SORIN, AND S. ZAMIR (2015): Repeated Games. Econometric Society Monographs. Cambridge: Cambridge University Press. [615]

MilgROM, P. R., AND R. J. WebER (1985): "Distributional Strategies for Games With Incomplete Information," Mathematics of Operations Research, 10, 619-632. [606,622]

Neyman, A., AND S. SORIN (2003): Stochastic Games and Applications. NATO Science Series: Mathematical and Physical Sciences. Dordrecht: Springer Verlag. [595,615]

NiElSEN, L. T. (1984): "Common Knowledge, Communication, and Convergence of Beliefs," Mathematical Social Sciences, 8 (1), 1-14. [606]

PARTHASARATHY, T. (1972): Selection Theorems and Their Applications. Lecture Notes in Mathematics. Berlin: Springer Verlag. [616]

(1973): "Discounted, Positive, and Noncooperative Stochastic Games," International Journal of Game Theory, 2 (1), 25-37. [612,613]

PeLEG, B. (1969): "Equilibrium Points for Games With Infinitely Many Players," Journal of the London Mathematical Society, 44 (1), 292-294. [619]

SimON, R. S. (2000): “The Common Prior Assumption in Belief Spaces: An Example,” Technical Report, Center For The Study of Rationality, Hebrew University of Jerusalem. [594,606]

(2003): "Games of Incomplete Information, Ergodic Theory, and the Measurability of Equilibria," Israel Journal of Mathematics, 138 (1), 73-92. [594,606-608,610]

Simon, R. S., AND G. TOMKOWICZ (2018): "A Bayesian Game Without $\epsilon$-Equilibria,” Israel Journal of Mathematics, 227 (1), 215-231. [594]

StinchсомBE, M. B. (2011): "Balance and Discontinuities in Infinite Games With Type-Dependent Strategies," Journal of Economic Theory, 146 (2), 656-671. [622] 
Sullivan, D., B. WeISS, AND J. Wright (1986): "Generic Dynamics and Monotone Complete C*-Algebras," Transactions of the American Mathematical Society, 295, 795-809. [623]

WAGNER, D. H. (1977): "Survey of Measurable Selection Theorems," SIAM Journal on Control and Optimization, 15 (5), 859-903. [626]

Co-editor Joel Sobel handled this manuscript.

Manuscript received 30 June, 2017; final version accepted 31 October, 2018; available online 7 November, 2018. 